\title{
Unveiling radio halos in galaxy clusters in the LOFAR era
}

\author{
R. Cassano ${ }^{1}$, G. Brunetti ${ }^{1}$, H. J. A. Röttgering ${ }^{2}$, and M. Brüggen ${ }^{3}$ \\ 1 INAF - Istituto di Radioastronomia, via P. Gobetti 101,I-40129 Bologna, Italy \\ e-mail: rcassano@ira.inaf.it \\ 2 Leiden Observatory, Leiden University, Oort Gebouw, PO Box 9513, 2300 RA Leiden, The Netherlands \\ 3 Jacobs University Bremen, PO Box 750 651, 28725, Bremen, Germany
}

Received 4 August 2009 / Accepted 7 October 2009

\section{ABSTRACT}

\begin{abstract}
Aims. Giant radio halos are mega-parsec scale synchrotron sources detected in a fraction of massive and merging galaxy clusters. Radio halos provide one of the most important pieces of evidence of non-thermal components in large-scale structure. Statistics of their properties can be used to discriminate among various models for their origin. Therefore, theoretical predictions of the occurrence of radio halos are important as several new radio telescopes are about to begin to survey the sky at low frequencies with unprecedented sensitivity.

Methods. We carry out Monte Carlo simulations to model the formation and evolution of radio halos in a cosmological framework. In the context of the turbulent re-acceleration model, we extend previous work on the statistical properties of radio halos.

Results. We first compute the fraction of galaxy clusters that show radio halos and derive the luminosity function of the radio halos. We then derive differential and integrated number count distributions of radio halos at low radio frequencies to explore the potential of the upcoming LOFAR surveys. By restricting ourselves to clusters at redshifts $<0.6$, we find that the planned LOFAR all-sky survey at $120 \mathrm{MHz}$ is expected to detect about 350 giant radio halos. About half of these halos have spectral indices greater than 1.9 and brighten substantially at lower frequencies. If detected they will enable us to confirm that turbulence accelerates the emitting particles. We also propose that commissioning surveys, such as $\mathrm{MS}^{3}$, have the potential to detect about 60 radio halos in clusters of the ROSAT brightest cluster sample and its extension (eBCS). These surveys will allow us to constrain how the rate of formation of radio halos in these clusters depends on cluster mass.
\end{abstract}

Key words. radiation mechanism: non-thermal - galaxies: clusters: general - radio continuum: general - X-rays: general

\section{Introduction}

Radio halos are diffuse Mpc-scale radio sources observed at the center of $\sim 30 \%$ of massive galaxy clusters (e.g., Feretti 2005; Ferrari et al. 2008, for reviews). These sources emit synchrotron radiation produced by $\mathrm{GeV}$ electrons diffusing through $\mu \mathrm{G}$ magnetic fields and provide the most important evidence of nonthermal components in the intra-cluster medium (ICM).

Clusters hosting radio halos always display evidence of very recent or ongoing merger events (e.g., Buote 2001; Schuecker et al. 2001; Govoni et al. 2004; Venturi et al. 2008). This suggests a connection between the gravitational process of cluster formation and the origin of these halos. Cluster mergers are expected to be the most important sources of non-thermal components in galaxy clusters. A fraction of the energy dissipated during these mergers could be channelled into amplification of the magnetic fields (e.g., Dolag et al. 2002; Brüggen et al. 2005; Subramanian et al. 2006; Ryu et al. 2008) and into the acceleration of high energy particles by shocks and turbulence (e.g., Enßlin et al. 1998; Sarazin 1999; Blasi 2001; Brunetti et al. 2001, 2004; Petrosian 2001; Miniati et al. 2001; Fujita et al. 2003; Ryu et al. 2003; Hoeft \& Brüggen 2007; Brunetti \& Lazarian 2007; Pfrommer et al. 2008; Brunetti et al. 2009).

A promising scenario proposed to explain the origin of the synchrotron emitting electrons in radio halos assumes that electrons are re-accelerated by the interaction with MHD turbulence injected into the ICM in connection with cluster mergers (turbulent re-acceleration model, e.g., Brunetti et al. 2001; Petrosian 2001). An alternative possibility is that the emitting electrons are continuously injected by $p p$ collisions in the ICM (secondary models; e.g., Dennison 1980; Blasi \& Colafrancesco 1999).

In the picture of the turbulent re-acceleration scenario, the formation and evolution of radio halos are tightly connected with the dynamics and evolution of the hosting clusters. Indeed, the occurrence of radio halos at any redshift depends on the rate of cluster-cluster mergers and on the fraction of the merger energy channelled into MHD turbulence and re-acceleration of high energy particles. In the past few years, this has been modeled by Monte Carlo procedures (Cassano \& Brunetti 2005; Cassano et al. 2006a) that provide predictions verifiable by future instruments. In this scenario radio halos have a relatively short lifetime $(\approx 1 \mathrm{Gyr})$, and the fraction of galaxy clusters in which radio halos are generated is expected to increase with cluster mass (or X-ray luminosity), since the energy of the turbulence generated during cluster mergers is expected to scale with the cluster thermal energy (which scales roughly as $\sim M^{5 / 3}$; e.g., Cassano \& Brunetti 2005). It has been shown that the predicted occurrence of radio halos as a function of the cluster mass (or X-ray luminosity) is in line with results obtained from a large observational project, the "GMRT radio halo survey" (Venturi et al. 2007, 2008), and its combination with studies of nearby halos based on the NVSS survey (e.g., Cassano et al. 2008).

The steep spectrum of radio halos makes these sources ideal targets for observations at low radio frequencies suggesting that present radio telescopes can only detect the tip of the iceberg of their population (Enßlin \& Röttgering 2002; Cassano et al. 2006a; Hoeft et al. 2008). The discovery of the giant and 
ultra-steep spectrum radio halo in Abell 521 at low radio frequencies (Brunetti et al. 2008) allows a first confirmation of this conjecture and provides a glimpse of what future low frequency radio telescopes, such as the Low Frequency Array (LOFAR) ${ }^{1}$ and the Long Wavelength Array (LWA, e.g., Ellingson et al. 2009), might find in upcoming years.

LOFAR promises an impressive gain of two orders of magnitude in sensitivity and angular resolution over present instruments in the frequency range $15-240 \mathrm{MHz}$, and as such will open up a new observational window to the Universe. In particular, LOFAR is expected to contribute significantly to the understanding of the origin and evolution of the relativistic matter and magnetic fields in galaxy clusters.

The main focus of the present paper is to provide a theoretical framework for the interpretation of future LOFAR data by quantifying expectations for the properties and occurrence of giant radio halos in the context of the turbulent re-acceleration scenario. In particular, in Sect. 2 we summarize the main ingredients used in the model calculations and provide an extension of the results of previous papers on the occurrence of radio halos in clusters (Sect. 2.1) and on the expected radio halo luminosity functions (Sect. 2.2). In Sect. 3, we derive the expected number counts of radio halos at $120 \mathrm{MHz}$ and explore the potential of LOFAR surveys. Our conclusions are given in Sect. 4.

$\operatorname{A} \Lambda \operatorname{CDM}\left(H_{\mathrm{o}}=70 \mathrm{Km} \mathrm{s}^{-1} \mathrm{Mpc}^{-1}, \Omega_{\mathrm{m}}=0.3, \Omega_{\Lambda}=0.7\right)$ cosmology is adopted throughout the paper.

\section{Statistical modelling of giant radio halos in galaxy clusters}

Turbulence generated during cluster mergers may accelerate relativistic particles and produce diffuse synchrotron emission from Mpc regions in galaxy clusters (e.g., Brunetti et al. 2008). Diffuse radio emission in the form of giant radio halos should be generated in connection with massive mergers and fade away as soon as turbulence is dissipated and the emitting electrons cool due to radiative losses. It is likely that the generation of turbulence and the acceleration of particles persist for a few crossing times of the cluster-core regions, implying a lifetime of about 1 Gyr.

Since the physics of the proposed scenario is rather uncertain, we choose to model the properties of the halos and their cosmic evolution using a simple statistical approach. By means of Monte Carlo calculations, we take into account the main processes that play a role in this scenario. These include the rate of cluster-cluster mergers in the Universe and their mass ratios, and the fraction of the energy dissipated during these mergers that is channelled into MHD turbulence and acceleration of high energy particles (Cassano \& Brunetti 2005; Cassano et al. 2006a). We refer the reader to these papers for details, here we briefly report the essential steps that enter into the calculations:

i) The formation and evolution of galaxy clusters is computed by the extended Press \& Schechter approach (1974, hearafter PS; Lacey \& Cole 1993), which is based on the hierarchical theory of cluster formation. The PS mass function shows good agreement with that derived from $N$-body simulations, at least for relatively low redshifts and masses $\sim 10^{14}-10^{15} \mathrm{~h}^{-1} M_{\odot}$ (e.g., Springel et al. 2005), although it has the tendency to underestimate the number density of systems with mass $\geq 10^{15} \mathrm{~h}^{-1} M_{\odot}$ (e.g., Governato et al. 1999;

\footnotetext{
1 http://www. lofar.org
}

Bode et al. 2001; Jenkins et al. 2001). Given the presentday mass and temperature of the parent clusters, the cluster merger history (merger trees) is obtained by using Monte Carlo simulations. We simulate the formation history of $\sim 1000$ galaxy clusters with present-day masses in the range $2 \times 10^{14}-6 \times 10^{15} M_{\odot}$. This allows a statistical description of the cosmological evolution of galaxy clusters and of the merging events with cosmic time.

ii) The generation of the turbulence in the ICM is estimated for each merger identified in the merger trees. The resulting turbulence is assumed to be generated and then dissipated within a timescale of the order of the cluster-cluster crossing time in that merger ${ }^{2}$. Furthermore, it is assumed that turbulence is generated in the volume swept by the subcluster infalling into the main cluster and that a fraction, $\eta_{t}$, of the $P \mathrm{~d} V$ work done by this subcluster goes into the excitation of fast magneto-acoustic waves. The $P \mathrm{~d} V$ work is estimated to be $\approx \rho v_{\mathrm{i}}^{2} \pi r_{\mathrm{s}}^{2} R_{\mathrm{v}}$, where $\rho$ is the ICM density of the main cluster averaged over the swept cylinder, $v_{\mathrm{i}}$ is the impact velocity of the two subclusters, $r_{\mathrm{s}}$ is the stripping radius (see also Sect. 2.1), and $R_{\mathrm{v}}$ is the virial radius of the main cluster (see Cassano \& Brunetti 2005, for details).

iii) The resulting spectrum of MHD turbulence generated by the chain of mergers in any synthetic cluster and its evolution with cosmic time is computed by taking into account the injection of waves and their damping in a collisionless plasma. Acceleration of particles by this turbulence and their evolution is computed in connection with the evolution of synthetic clusters by solving Fokker-Planck equations and including the relevant energy losses.

iv) This procedure allows for the exploration of the statistical properties of radio halos. Following Cassano et al. (2006a), we consider homogeneous models, i.e., without spatial variation in the turbulent energy, acceleration rate, and magnetic field in the halo volume. We assume a value of the magnetic field, averaged over a region of radius $R_{\mathrm{H}} \simeq 500 \mathrm{~h}_{50}^{-1} \mathrm{kpc}$, which scales with the virial mass of clusters, $M_{\mathrm{v}}$ as

$\langle B\rangle=B_{\langle M\rangle}\left(\frac{M_{\mathrm{v}}}{\langle M\rangle}\right)^{b}$,

where $b>0$ is a parameter that enters into the model calculations. Equation (1) is motivated by numerical cosmological (MHD) simulations that found a scaling of the magnetic field with temperature or mass of the simulated clusters (e.g., Dolag et al. 2002) ${ }^{3}$.

\subsection{Occurrence of radio halos in galaxy clusters}

Stochastic particle acceleration by MHD turbulence is believed to be rather inefficient in the ICM. Consequently, electrons can be accelerated only up to energies of $m_{\mathrm{e}} c^{2} \gamma_{\max } \leq$ several $\mathrm{GeV}$, since at higher energies the radiation losses are efficient and hence dominate. The resulting gradual steepening in the theoretical synchrotron spectrum of radio halos at high frequencies is consistent with the observed spectral shapes (or with the very steep spectra) of several well studied halos (e.g., Schlickeiser et al. 1987; Thierbach et al. 2003; Brunetti et al. 2008; Dallacasa et al. 2009).

\footnotetext{
2 The cascading timescale of large-scale turbulence is expected to be of the same order as the cluster-cluster crossing time (e.g., Cassano \& Brunetti 2005; Brunetti \& Lazarian 2007).

3 Dolag et al. (2002) found a scaling $B \propto T^{2}$, which would imply that $B \propto M^{4 / 3}$ if the virial scaling $M \propto T^{3 / 2}$ is assumed.
} 
This steepening makes it difficult to detect these sources at frequencies higher than the frequency, $v_{\mathrm{s}}$, at which the steepening becomes severe (see Fig. 1), where $v_{\mathrm{S}}$ is expected to be a few times higher than the break frequency, $v_{\mathrm{b}}$, and $v_{\mathrm{b}}$ depends on the acceleration efficiency in the ICM, $\chi$, being defined by (e.g., Cassano et al. 2006a)

$\nu_{\mathrm{b}} \propto\langle B\rangle \gamma_{\max }^{2} \propto \frac{\langle B\rangle \chi^{2}}{\left(\langle B\rangle^{2}+B_{\mathrm{cmb}}^{2}\right)^{2}}$.

The transit time damping (TTD) is the most important collisionless resonance between the magnetosonic waves and particles, and is produced by the interaction of the compressible component of the magnetic field of these waves with the particles (e.g., Schlickeiser \& Miller 1998; Cassano \& Brunetti 2005; Brunetti \& Lazarian 2007). In this case $\chi \simeq 4 D_{\mathrm{pp}} / p^{2}$, where $p$ is the momentum of the electrons and $D_{\mathrm{pp}}$ is the electron diffusion coefficient in the momentum space due to the coupling with turbulent waves. In the case of a single merger between a cluster with mass $M_{\mathrm{v}}$ and a subcluster of mass $\Delta M$, Cassano \& Brunetti (2005) derived that $\chi$ can be approximated by

$\chi \propto \frac{\eta_{t}}{R_{\mathrm{H}}^{3}}\left(\frac{M_{\mathrm{v}}+\Delta M}{R_{\mathrm{v}}}\right)^{3 / 2} \frac{r_{\mathrm{s}}^{2}}{\sqrt{k_{B} T}} \times \begin{cases}1 & \text { if } r_{\mathrm{s}} \leq R_{\mathrm{H}} \\ \left(R_{\mathrm{H}} / r_{\mathrm{s}}\right)^{2} & \text { if } r_{\mathrm{s}}>R_{\mathrm{H}},\end{cases}$

where $r_{\mathrm{s}}$ is the stripping radius of the subcluster crossing the main cluster, i.e., the distance from the center of the subcluster where the static pressure equals the ram pressure (see Cassano \& Brunetti 2005 for details), $R_{\mathrm{H}}$ is the size of the radio halo, and $R_{\mathrm{V}}$ and $T$ are the virial radius and temperature of the main cluster, respectively.

Combined with Eq. (2), this implies that higher values of $v_{\mathrm{b}}$ are expected in the more massive clusters, $\nu_{\mathrm{b}} \propto\left(M_{\mathrm{v}} / R_{\mathrm{v}}\right)^{3} / T \propto$ $M_{\mathrm{v}}^{4 / 3}$ (here considering for simplicity a fixed value of $B$, see Cassano et al. 2006a for a more general discussion), and in connection with major merger events, $v_{\mathrm{b}} \propto(1+\Delta M / M)^{3}\left(r_{\mathrm{s}}\right.$ in Eq. (3) also increases with $\Delta M / M$ ).

Monte Carlo simulations can now be used to follow clustermergers and to explore how different mergers contribute to the acceleration (efficiency) of relativistic particles in the ICM. Consequently, this allows a statistical modeling of $v_{\mathrm{b}}$ to be performed within a synthetic cluster sample and the derivation of its statistical dependence on cosmic time and cluster mass.

Surveys cannot detect radio halos that have $v_{\mathrm{S}}$ lower than the observing frequency, since the spectrum of these halos should be very steep and their emission should fall below the survey detection limit (Fig. 1). To investigate the statistical behavior of the population of radio halos at different frequencies, we only consider halos to be observable when $v_{\mathrm{s}} \geq v_{\mathrm{o}}$. Figure 2 shows the ratio $v_{\mathrm{s}} / v_{\mathrm{b}}$ calculated for homogeneous models of radio halos, defining $v_{\mathrm{s}}$ as the frequency where the synchrotron spectrum of these halos is $\alpha=1.9$ ( $\alpha=1.9$ being calculated between $v_{\mathrm{s}} / 2.5$ and $v_{\mathrm{s}}$ to mimic $600-1400 \mathrm{MHz}$ spectra); since $v_{\mathrm{s}} / v_{\mathrm{b}}$ is only mildly dependent on magnetic field strength and the assumed fraction of turbulent energy injected, we adopt $v_{\mathrm{s}} \sim 7 v_{\mathrm{b}}$. A statistical modeling of $v_{\mathrm{b}}$ provides a statistical evaluation of $v_{\mathrm{s}}$ in the synthetic cluster sample.

In the context of the turbulent acceleration model for giant radio halos, energetics arguments imply that halos with $v_{\mathrm{s}} \geq$ $1 \mathrm{GHz}$ must be generated in connection with the most energetic merger-events in the Universe. Only these mergers can produce the efficient acceleration necessary to have relativistic electrons emitting at these frequencies (Cassano \& Brunetti 2005). Present surveys carried out at $v_{\mathrm{o}} \sim 1 \mathrm{GHz}$ detect radio halos only in the

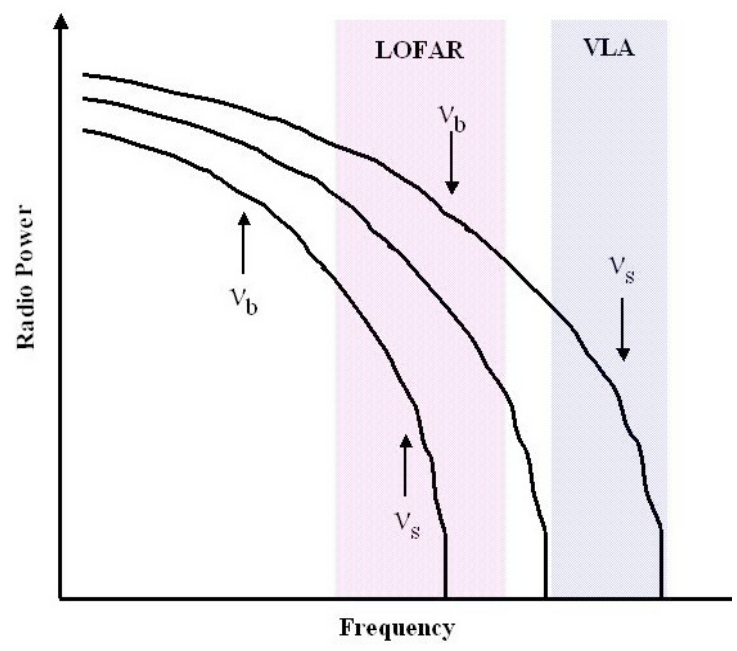

Fig. 1. A schematic representation of the theoretical synchrotron spectra of radio halos with different values of $v_{\mathrm{s}}$ (and $v_{\mathrm{b}}$ ). The colored regions indicate the frequency range of VLA and LOFAR observations.

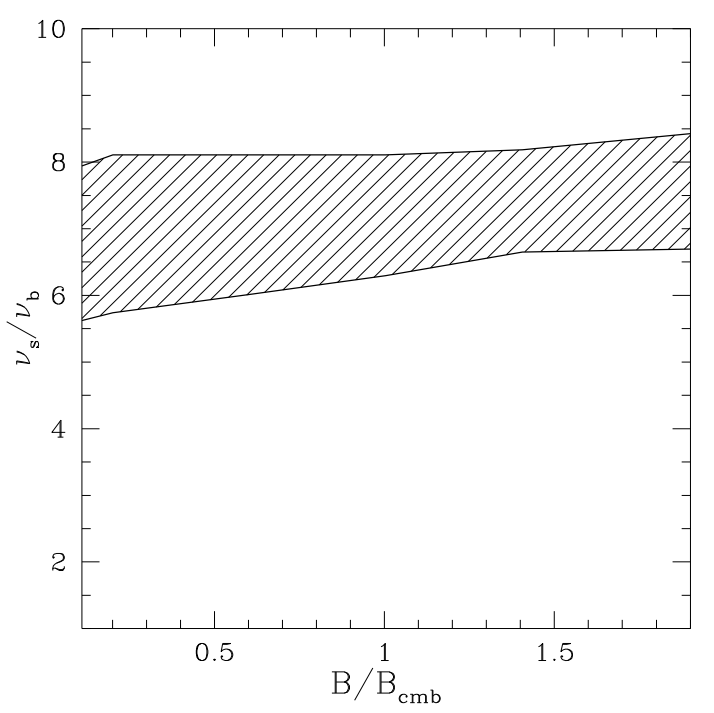

Fig. 2. Ratio $v_{\mathrm{s}} / v_{\mathrm{b}}$ as a function of $B / B_{\mathrm{cmb}}$ calculated with homogeneous models. Particle acceleration is calculated using TTD resonance with magneto-acoustic waves (Brunetti \& Lazarian 2007). The shadowed region marks the range of $v_{\mathrm{s}} / v_{\mathrm{b}}$ obtained assuming different energy density of magneto-acoustic turbulence, $3 \%-30 \%$ of the thermal energy density, and duration of the acceleration process, $\Delta t=2-4 \times \tau_{\text {acc }}$, where the acceleration timescale is $\tau_{\mathrm{acc}}=\chi^{-1}=p^{2} /\left(4 D_{\mathrm{pp}}\right)$.

most massive and merging clusters (e.g., Buote 2001; Venturi et al. 2008), and their occurrence has been used to constrain the value of $\eta_{t} \approx 0.1-0.3$ in the models (Cassano \& Brunetti 2005). Similar energetics arguments can be used to claim that radio halos with lower values of $v_{\mathrm{s}}$ must be more common, since they can be generated in connection with less energetic phenomena, e.g., major mergers between less massive systems or minor mergers in massive systems (e.g., Eqs. (2)-(3)), that are more common in the Universe (e.g., Cassano et al. 2008).

In Fig. 3, we plot the fraction of clusters that host radio halos with $v_{\mathrm{s}} \geq v_{\mathrm{o}}$ as a function of the cluster mass and by considering two redshift ranges : 0-0.1 (left panel) and 0.4-0.5 (right panel); this is obtained by assuming a reference set of model parameters, namely $\langle B\rangle=1.9 \mu \mathrm{G}, b=1.5, \eta_{t}=0.2$ (see also Cassano et al. 2006a). As expected, the fraction of clusters with halos increases at lower values of $v_{0}$, and the size of this increment depends on 

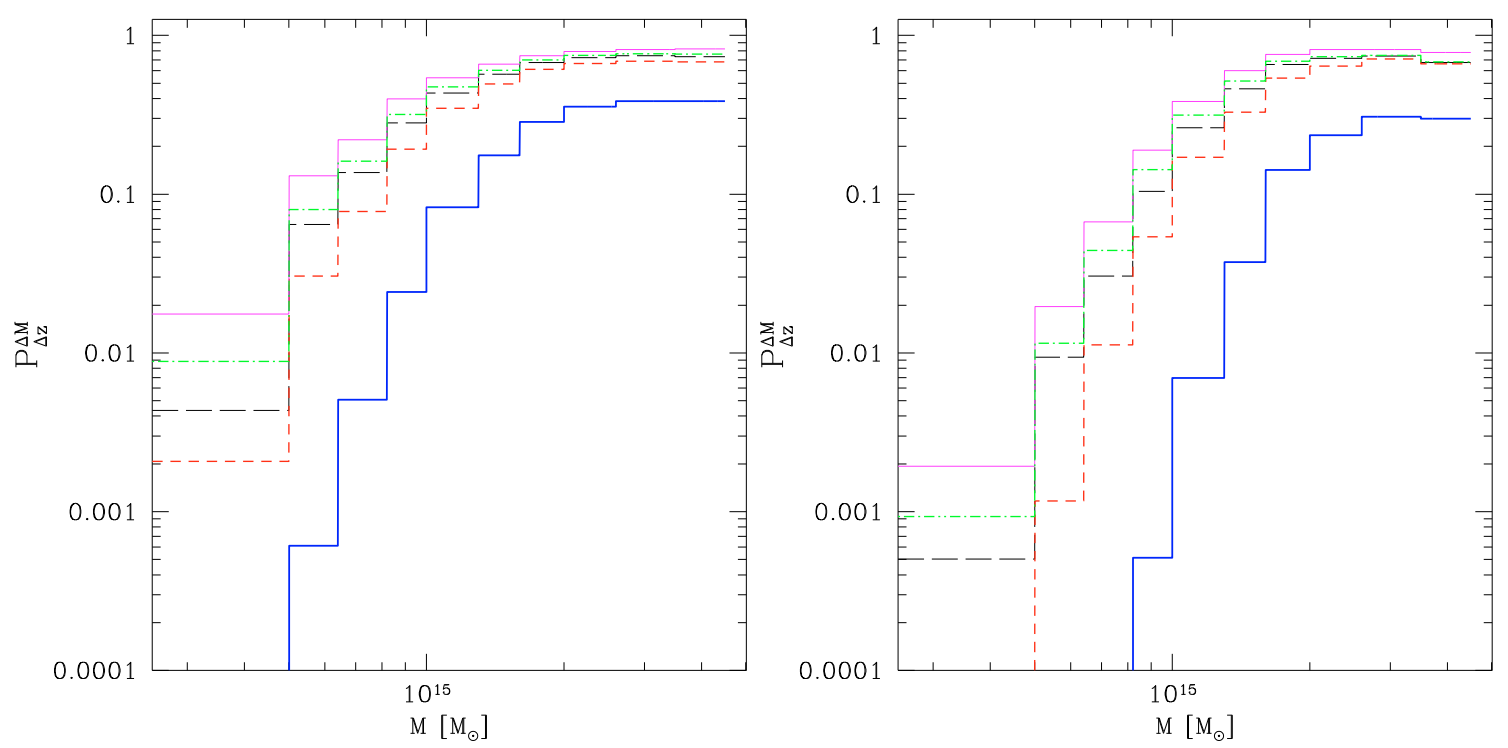

Fig. 3. Fraction of clusters with radio halos, with $v_{\mathrm{s}} \geq v_{\mathrm{o}}$, as a function of the cluster mass in the redshift range 0-0.1 (left panel) and 0.4-0.5 (right panel). Calculations assume $v_{\mathrm{o}}=1.4 \mathrm{GHz}, 240 \mathrm{MHz}, 150 \mathrm{MHz}, 120 \mathrm{MHz}$, and $74 \mathrm{MHz}$ (from bottom to top).
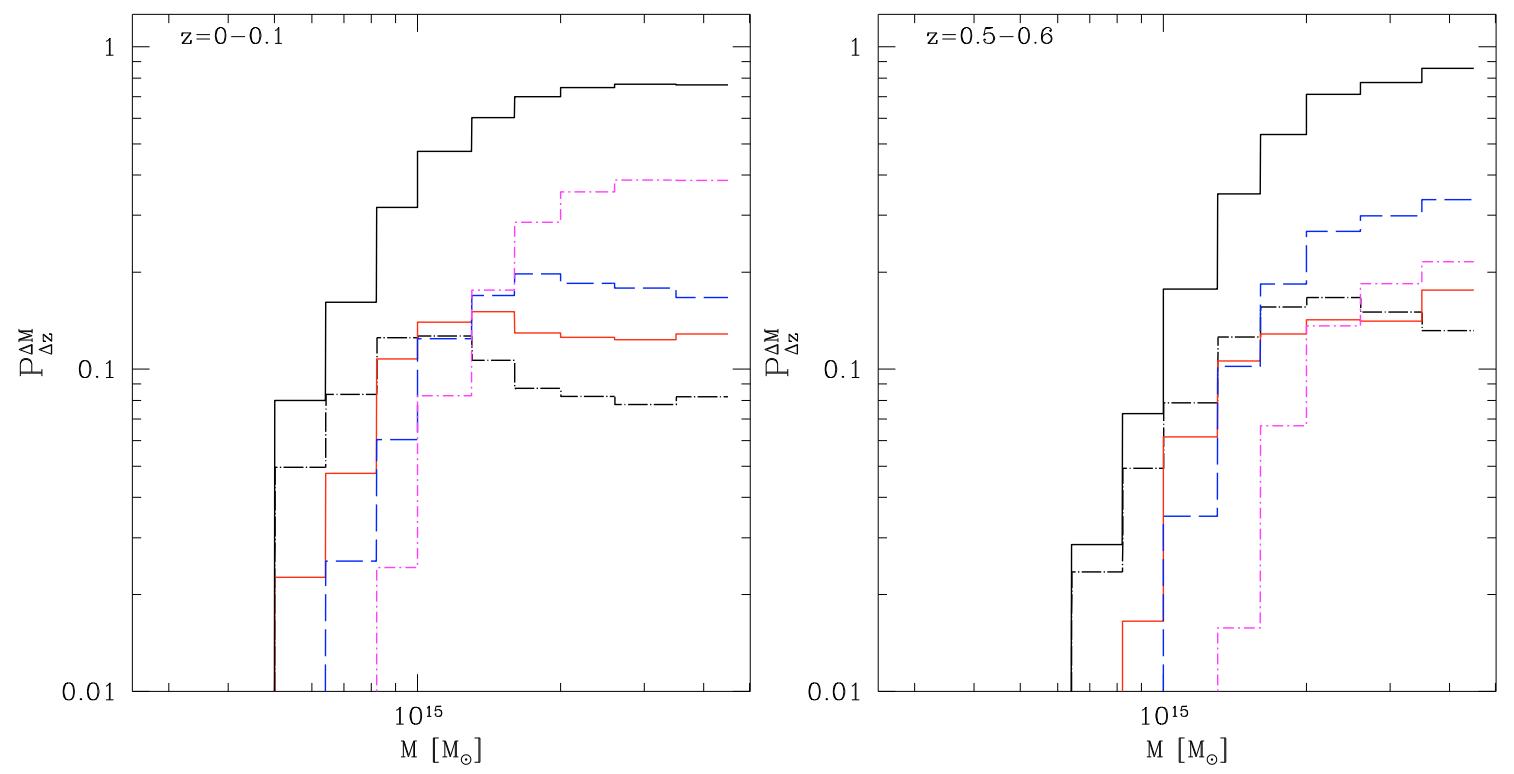

Fig. 4. Fraction of clusters with radio halos with $v_{\mathrm{s}} \geq 120 \mathrm{MHz}$ (black, upper, solid lines) as a function of the cluster mass in the redshift range $0-0.1$ (left panel) and 0.5-0.6 (right panel). The fractions of clusters with radio halos $v_{\mathrm{s}}$ in different frequency ranges are also shown: $v_{\mathrm{s}} \geq 1400 \mathrm{MHz}, 600<v_{\mathrm{s}}<1400 \mathrm{MHz}, 240<v_{\mathrm{s}}<600 \mathrm{MHz}$, and $120<v<240 \mathrm{MHz}$ (from top to bottom).

the considered mass and redshift of the parent clusters, being greater at lower cluster masses and at higher redshifts.

In Fig. 4, we plot the fraction of radio halos with $v_{\mathrm{s}} \geq$ $120 \mathrm{MHz}$ (black upper line) and the differential contribution to this fraction from radio halos with $v_{\mathrm{s}}$ in four frequency ranges (see figure caption for details). For nearby systems (Fig. 4, Left Panel), a significant fraction of massive clusters, $M_{\mathrm{v}}>10^{15} M_{\odot}$, is expected to host radio halos with $v_{\mathrm{s}} \geq 120 \mathrm{MHz}$; a sizeable fraction of them with $v_{\mathrm{s}}>600 \mathrm{MHz}$ (blue and magenta lines). On the other hand, the majority of radio halos in clusters with mass $M_{\mathrm{v}} \lesssim 10^{15} M_{\odot}$ would have very steep spectra if observed at $\mathrm{GHz}$ frequencies, $v_{\mathrm{s}}<600 \mathrm{MHz}$ (red line and black dot-dashed line). Our calculations suggest that a similar situation is expected for clusters at higher redshift (Fig. 4, right panel). Radio halos with higher values of $v_{\mathrm{s}}$ become much rarer with increasing redshift, mainly because the unavoidable inverse Compton losses at these redshifts limit the maximum energy of the accelerated electrons in these systems. At $z>0.5$, only merging clusters with mass $M_{\mathrm{v}} \gtrsim 2 \times 10^{15} M_{\odot}$ have a sizeable chance of hosting giant radio halos with $v_{\mathrm{s}} \geq 1.4 \mathrm{GHz}$, and an increasing contribution to the percentage of radio halos at higher redshift comes from halos with lower $v_{\mathrm{s}}$.

\subsection{The radio halo luminosity function}

The luminosity functions of radio halos (RHLFs), i.e., the number of halos per comoving volume and radio power, with $v_{\mathrm{s}} \geq$ 1.4 GHz was derived by Cassano et al. (2006a) to be

$$
\frac{\mathrm{d} N_{\mathrm{H}}(z)}{\mathrm{d} V \mathrm{~d} P(1.4)}=\frac{\mathrm{d} N_{\mathrm{H}}(z)}{\mathrm{d} M \mathrm{~d} V} / \frac{\mathrm{d} P(1.4)}{\mathrm{d} M}
$$


where $\mathrm{d} N_{\mathrm{H}}(z) / \mathrm{d} M \mathrm{~d} V$ is the theoretical mass function of radio halos with $v_{\mathrm{s}} \geq 1.4 \mathrm{GHz}$, that is obtained by combining Monte Carlo calculations of the fraction of clusters with halos and the PS mass function of clusters (e.g., Eq. (18) in Cassano et al. 2006a).

The quantity $\mathrm{d} P(1.4) / \mathrm{d} M$ can be estimated from the correlation between the $1.4 \mathrm{GHz}$ radio power, $P(1.4)$, and the mass of the parent clusters that is observed for radio halos (e.g., Govoni et al. 2001; Cassano et al. 2006a). Cassano et al. (2006a) discussed the $P(1.4)-M_{\mathrm{v}}$ correlation in the context of the turbulent acceleration model and demonstrated that the slope is consistent with the observed value $\left(\alpha_{M}=2.9 \pm 0.4\right)$ for a well constrained region of parameter space $\left(B_{\langle M\rangle}, b\right.$, and $\eta_{t}$; Fig. 7 in Cassano et al. 2006a); model parameters adopted in the present paper, i.e., $\langle B\rangle=1.9 \mu \mathrm{G}, b=1.5$ and $\eta_{t}=0.2$, fall in this range. In particular, the value of the derivative $\mathrm{d} P(1.4) / \mathrm{d} M$ in Eq. (4) depends on the set of parameters $\left(B_{\langle M\rangle}, b\right)$ that, in the case of the reference model we use in this paper, sets $\alpha_{M}=3.3$.

To derive the RHLF at frequency $v_{0}$, the contribution of all radio halos with $v_{\mathrm{S}} \geq v_{\mathrm{O}}$ should be taken into account. We first obtained the RHLF for halos with $v_{\mathrm{s}}$ in a given frequency interval, e.g., $\Delta v_{\mathrm{si}}$, and then combined the different contributions from the considered intervals $\Delta v_{\mathrm{si}}$ :

$\frac{\mathrm{d} N_{\mathrm{H}}(z)}{\mathrm{d} V \mathrm{~d} P\left(v_{\mathrm{o}}\right)}=\sum_{\mathrm{i}}\left(\frac{\mathrm{d} N_{\mathrm{H}}(z)}{\mathrm{d} M \mathrm{~d} V}\right)_{\Delta v_{\mathrm{si}}}\left(\frac{\mathrm{d} P\left(v_{\mathrm{o}}\right)}{\mathrm{d} M}\right)_{\Delta v_{\mathrm{si}}}^{-1}$.

To derive the contribution to the RHLF from radio halos with $v_{\mathrm{s}} \geq 1.4 \mathrm{GHz}$, we should calculate $\mathrm{d} P\left(v_{\mathrm{o}}\right) / \mathrm{d} M$ for these halos. This can be estimated from the $P(1.4)-M_{\mathrm{v}}$ correlation assuming a monochromatic radio power of these halos at $v_{\mathrm{o}}$ given by

$P_{1.4}\left(v_{\mathrm{o}}, M_{\mathrm{v}}\right)=P_{1.4}\left(1.4, M_{\mathrm{v}}\right)\left(\frac{1400 \mathrm{MHz}}{v_{\mathrm{o}}}\right)^{\alpha}$,

where $P_{1.4}\left(1.4, M_{\mathrm{v}}\right)$ is the monochromatic radio power at $1.4 \mathrm{GHz}$ from the $P(1.4)-M_{\mathrm{v}}$ correlation, and $\alpha \sim 1.3$ is the typical spectral index of these halos, $P(v) \propto v^{-\alpha}$ (e.g., Ferrari et al. 2008).

We now consider the case of halos with $v_{\mathrm{s}}<1.4 \mathrm{GHz}$. The bolometric synchrotron power of radio halos is expected to scale with $v_{\mathrm{b}}$ and $B$ (e.g., Cassano et al. 2006a) such that

$P_{\text {syn }} \approx P\left(v_{\mathrm{b}}\right) v_{\mathrm{b}} \propto B v_{\mathrm{b}} \Rightarrow P\left(v_{\mathrm{b}}\right) \propto B$.

From Eqs. (2)-(3), it is clear that clusters of the same mass $M_{\mathrm{v}}$ (and magnetic field $B$ ) at redshift $z$ could have different values of $v_{\mathrm{b}}$, depending on the merger event responsible for the generation of the radio halo. Yet, for a fixed cluster mass (and consequently for a fixed value of the magnetic field), Eq. (7) implies that the synchrotron radio power emitted at the break frequency, $P\left(v_{\mathrm{b}}\right)$, is constant. In addition, homogeneous models, that consider an average value of $B$ and $\gamma_{\max }$ in the halo volume, also imply that $P\left(v_{\mathrm{b}}\right) v_{\mathrm{b}} \propto P\left(v_{\mathrm{s}}\right) v_{\mathrm{s}}$ (Fig. 2). From Eq. (7), we can then derive the monochromatic radio power at $v_{\mathrm{o}}$ of halos with a given $v_{\mathrm{s}}$ to be

$P_{v_{\mathrm{s}}}\left(v_{\mathrm{o}}, M_{\mathrm{v}}\right)=P_{v_{\mathrm{s}}}\left(v_{\mathrm{s}}, M_{\mathrm{v}}\right)\left(\frac{v_{\mathrm{s}}}{v_{\mathrm{o}}}\right)^{\alpha}=P_{1.4}\left(1.4, M_{\mathrm{v}}\right)\left(\frac{v_{\mathrm{s}}}{v_{\mathrm{o}}}\right)^{\alpha}$.

This allows the evaluation of $\left(\mathrm{d} P\left(v_{\mathrm{o}}\right) / \mathrm{d} M\right)_{\Delta v_{\mathrm{si}}}$ starting from $\mathrm{d} P(1.4) / \mathrm{d} M$, and thus the derivation of Eq. (5).

We also note that from Eqs. (6) and (8), one has

$P_{v_{\mathrm{s}}}\left(v_{\mathrm{o}}, M_{\mathrm{v}}\right)=P_{1.4}\left(v_{\mathrm{o}}, M_{\mathrm{v}}\right)\left(\frac{v_{\mathrm{s}}}{1400 \mathrm{MHz}}\right)^{\alpha}$,

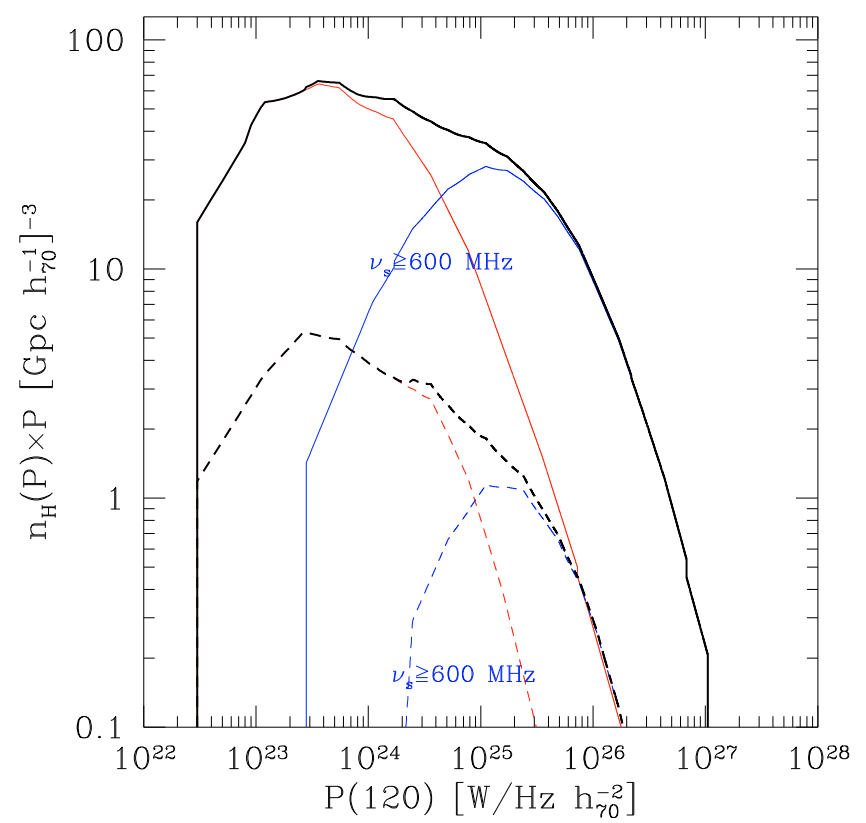

Fig. 5. Radio halo luminosity function at $v_{\mathrm{o}}=120 \mathrm{MHz}$ (black lines) for clusters at redshift $0-0.1$ (solid thick lines) and 0.5-0.6 (dashed thin lines). The contribution from halos with $120<v_{\mathrm{s}}<600 \mathrm{MHz}$ (red lines) and with $v_{\mathrm{s}} \geq 600 \mathrm{MHz}$ (blue lines) are also shown.

i.e., radio halos with synchrotron spectra that steepen at lower frequencies will also have monochromatic radio powers at $v_{\mathrm{o}}$ that are lower than those of radio halos of higher $v_{\mathrm{s}}$.

As a relevant example, in Fig. 5 we report the expected RHLF at $120 \mathrm{MHz}$ (black lines) for $z=0-0.1$ (solid thick lines) and $z=0.5-0.6$ (dashed thick lines), where we also show the relative contributions of halos with $v_{\mathrm{s}}<600 \mathrm{MHz}$ (red lines) and $v_{\mathrm{s}}>600 \mathrm{MHz}$ (blue lines).

As already discussed in Cassano et al. (2006a), the shape of the RHLF flattens at lower radio powers because of the decrease in the efficiency of particle acceleration in less massive clusters. We note that halos with $v_{\mathrm{s}}>600 \mathrm{MHz}$ (blue lines, Fig. 5) do not contribute to lower radio powers in the RHLF. This is because higher-frequency halos are generated in very energetic merger events, and must be extremely rare in smaller systems and consequently their monochromatic radio power is greater than that of halos with $v_{\mathrm{s}}<600 \mathrm{MHz}$ (red lines, Fig. 5). Finally, we note that with increasing redshift the RHLFs decrease due to the evolution with $z$ of both the cluster mass function and the fraction of galaxy clusters with radio halos (Fig. 3, see also Cassano et al. 2006a). The evolution of the RHLF with $z$ is stronger at higher radio powers, where the dominant contribution to the RHLF comes from halos with higher $v_{\mathrm{s}}$ and the fraction of clusters hosting these halos decreases more rapidly with redshift (e.g., Fig. 4).

\section{Number counts of radio halos and LOFAR surveys at $120 \mathrm{MHz}$}

It has been shown that model expectations of the occurrence of radio halos observed at $v_{0}=1.4 \mathrm{GHz}$ are consistent with the fraction of radio halos with cluster mass (Cassano et al. 2008) and with the number counts of nearby radio halos (Cassano et al. 2006a).

As already discussed, in this paper we adopt a reference model with parameters: $\langle B\rangle=1.9 \mu \mathrm{G}, b=1.5, \eta_{t}=0.2$. In Fig. 6, we report number counts of giant radio halos expected 


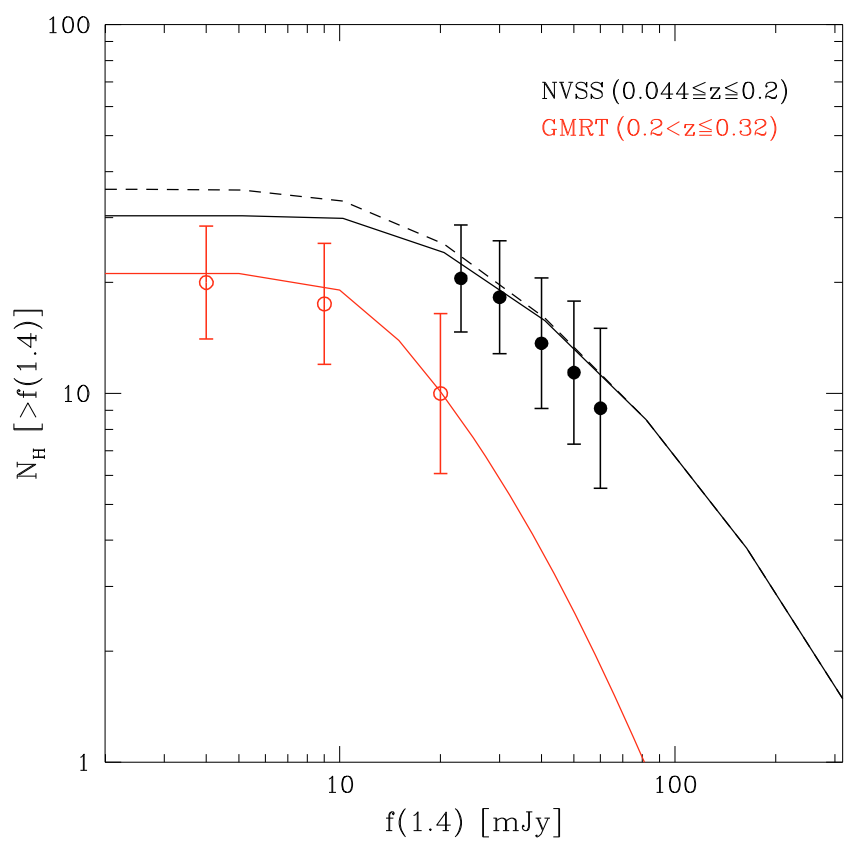

Fig. 6. All-sky integrated RHNCs for: (1) $z=0.044-0.2$, obtained by considering a minimum mass of clusters constrained at any $z$ by the XBACS X-ray flux limit (Ebeling et al. 1996) (dashed black line), and by combining the above mass-constraint with that implied by the NVSS sensitivity (following Cassano et al. 2008, see their Fig. 3) (solid upper black line); (2) $z=0.2-0.32$, obtained by considering the X-ray luminosity-range of the GMRT cluster sample (Venturi et al. 2007, 2008, red lower line). Black filled points are the observed RHNC of giant radio halos from NVSS-selected clusters in the redshift range $0.044-0.2$, re-normalized to account for the NVSS and XBACS sky coverage (and XBACS completeness). Red open points are the observed RHNC of giant radio halos in the GMRT cluster sample (with redshift $z=0.2-0.32$ ), re-normalized to account for the sky coverage of the GMRT cluster sample.

with these parameters compared with radio halo counts from the NVSS survey at low redshift, $0.044 \leq z \leq 0.2$ (Giovannini et al. 1999 ) and from the GMRT radio halo survey at intermediate redshift, $z=0.2-0.32$ (Venturi et al. 2007, 2008). The latter is a pointed survey down to $70 \mu \mathrm{Jy} / \mathrm{beam}$ at $610 \mathrm{MHz}$ of a sample of $\sim 50$ galaxy clusters extracted from the REFLEX (Böhringer et al. 2004) and eBCS (Ebeling et al. 1998, 2000) cluster catalogs. The clusters have $z=0.2-0.4$ and $L_{X[0.1-2.4 \mathrm{kev}]} \geq 5 \times$ $10^{44} \mathrm{erg} / \mathrm{s}$ (the X-ray sample is complete for $z \leq 0.32$; see Cassano et al. 2008). All halos in the survey have $1.4 \mathrm{GHz}$ follow-up data. Beside the fair agreement between expectations and observations (see caption), we note that the GMRT radio halo survey is sufficiently sensitive to detect relatively faint halos and constrain the flattening of the distribution of number counts of radio halos (RHNC) at lower fluxes.

Encouraged by these results, in this section we derive the expected RHNC at $120 \mathrm{MHz}$ and explore the potential of upcoming LOFAR surveys.

Because in our simplified procedure the radio power of halos scales with a spectral slope $\alpha=1.3$ (Eqs. (6)-(9)) and the vast majority of halos is at $z \sim 0.2-0.4$, in the following we neglect the $K$-correction ${ }^{4}$.

\footnotetext{
${ }^{4}$ For simplicity, we also consider as observables those halos with $v_{\mathrm{s}} \approx v_{\mathrm{o}}$ regardless of their redshift. This would slightly affect only the number counts of halos with $v_{\mathrm{s}} \leq v_{\mathrm{o}}(1+z)$ that represent a minimal fraction of our halo population.
}

\subsection{LOFAR surveys}

LOFAR will carry out surveys between $15 \mathrm{MHz}$ and $210 \mathrm{MHz}$ with unprecedented sensitivity and spatial resolution (e.g., Röttgering et al. 2006). The unprecedented ( $u, v)$ coverage of LOFAR on short baselines also maximizes the instrument capability to detect extended sources of low surface brightness such as radio halos. These surveys will constrain models of diffuse radio emission in galaxy clusters. In this paper, we assume an observing frequency $v_{\mathrm{o}}=120 \mathrm{MHz}$, at which LOFAR will carry out the deepest large-area radio surveys (e.g., Röttgering et al. 2006).

The crucial step in our analysis is the estimate of the minimum diffuse flux from giant radio halos (integrated over a scale of $\sim 1 \mathrm{Mpc}$ ) that is detectable by these surveys as a function of redshift. This depends on the brightness profiles of radio halos that is known to smoothly decrease with distance from the cluster center (e.g., Govoni et al. 2001). Consequently, the outermost, low brightness, regions of halos will be difficult to detect.

However what is important is the capability to detect the central, brightest, regions of radio halos in the survey images. Following Brunetti et al. (2007), we consider a shape of the radial profile of radio halos that is obtained from the analysis of well studied halos. We assume a circular observing beam $=25 \times 25$ arcsec, and follow two complementary approaches ${ }^{5}$ :

i) Since radio halos emit about half of their total radio flux within their half radius (Brunetti et al. 2007), we estimate the minimum flux of a detectable halo, $f_{\min }(z)$, by requiring that the mean brightness within $R_{\mathrm{H}} / 2, \frac{f_{\min } / 2}{\pi\left(\theta_{\mathrm{H}} / 2\right)^{2}}$, is $\xi$ times the rms, $F$, of the survey, i.e.,

$$
f_{\min }(z) \simeq 10^{-3}\left(\frac{\xi F}{0.5 \mathrm{mJy} / \text { beam }}\right) \theta_{\mathrm{H}}^{2}(z)[\mathrm{mJy}],
$$

where $\theta_{\mathrm{H}}(z)$ is the angular size of radio halos, in arcseconds, at a given redshift, allowing for the detection of diffuse halo emission in the images produced by the survey. Injection of fake radio halos in the $(u, v)$ plane of interferometric data from NVSS observations show that radio halos at $z \leq 0.3$ become visible in the images as soon as their flux approaches that obtained by Eq. (10) with $\xi \sim 1-2$ (Cassano et al. 2008).

ii) Following a second approach, we estimate the minimum flux of a detectable halo by requiring that the average brightness within 5 observing beams is $3 \times \xi$ times the rms, $F$, of the survey. The minimum flux is obtained from the condition

$2 \pi \int_{0}^{b_{\mathrm{s}}} I(b) b \mathrm{~d} b=5 S_{\text {beam }}(3 \xi F)$,

where $I(b)$ is the typical radial profile of halos (Brunetti et al. 2007), $S_{\text {beam }}$ is the beam area, and $b_{\mathrm{s}}=\left(5 S_{\text {beam }} / \pi\right)^{1 / 2}$. The aim of this second approach is to avoid any bias related to the redshift of the halos since, in the first approach, the sensitivity limit is reached across a fairly large area (many beams) for nearby radio halos, but only within an area of few beams in the case of halos at $z=0.5-0.6$.

Figure 7 shows $f_{\min }$ of radio halos as a function of redshift (left panel), and the corresponding minimum radio power (right panel), obtained following the two approaches and assuming that $\xi F=0.1,0.25,0.5,1,1.5 \mathrm{mJy} /$ beam (see figure caption).

5 The $120 \mathrm{MHz}$ LOFAR survey will have a full resolution of 5-6 arcsec, thus we are considering the case of tapered images that increase the sensitivity to extended emission without changing significantly the point source sensitivity (due to the large number of inner LOFAR stations). 
R. Cassano et al.: Unveiling radio halos in galaxy clusters in the LOFAR era
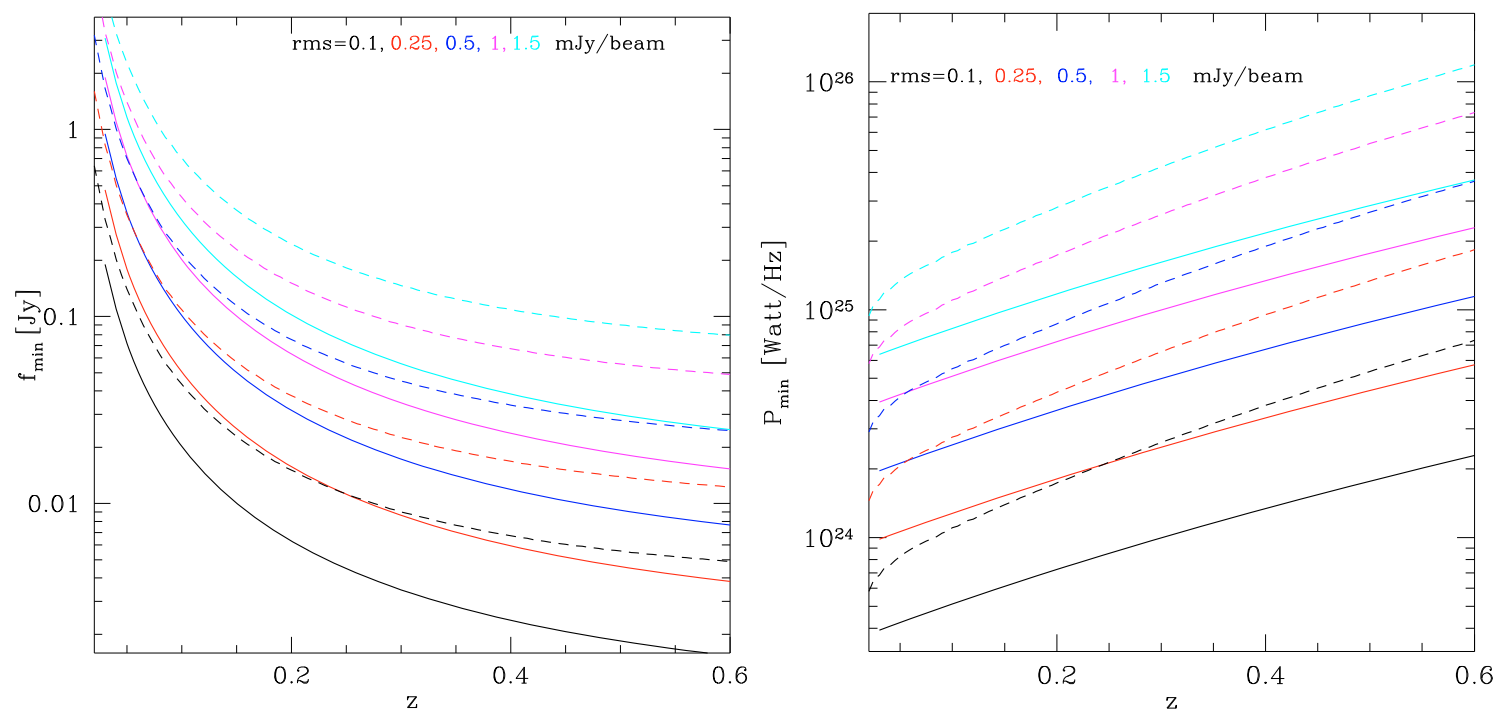

Fig. 7. Minimum flux (left panel) and power (right panel) of detectable radio halos at $120 \mathrm{MHz}$ using Eq. (10) (solid lines) and Eq. (11) (dashed lines), where $\xi F=0.1,0.25,0.5,1.0,1.5 \mathrm{mJy} / \mathrm{b}$ are assumed (from bottom to top), and beam $=25 \times 25$ arcsec.
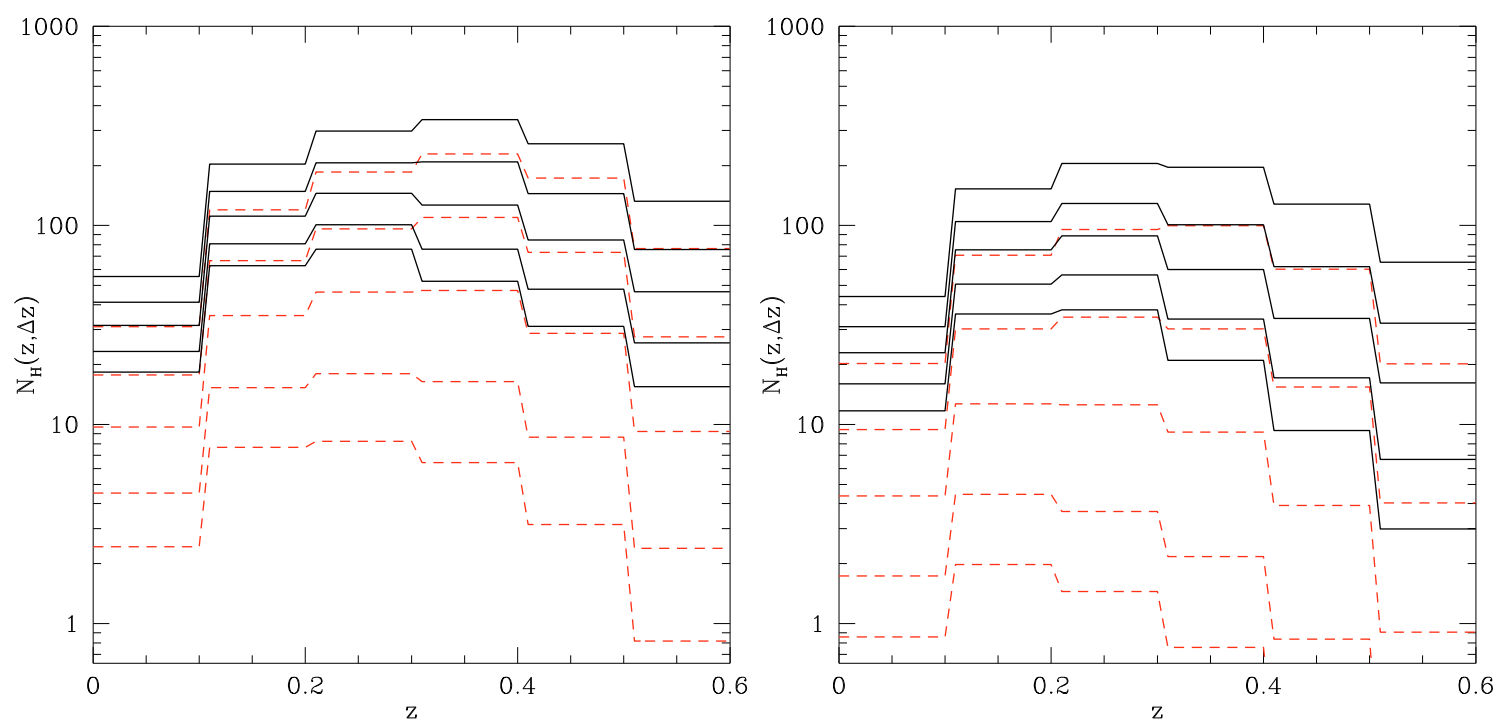

Fig. 8. Number (all-sky) of radio halos with $v_{\mathrm{s}} \geq 120 \mathrm{MHz}$ (black solid lines) as a function of redshift that can be expected at the sensitivity of LOFAR surveys. Calculations are performed following approaches i) in Sect. 2.1 (left); and ii) (right), assuming $\xi \mathrm{rms}=0.1,0.25,0.5,1.0,1.5$ (bottom to top). Red dashed lines give the number counts of radio halos with $120 \leq v_{\mathrm{s}} \leq 600 \mathrm{MHz}$.

Given the RHLF $\left(\mathrm{d} N_{\mathrm{H}}(z) / \mathrm{d} P\left(v_{\mathrm{o}}\right) \mathrm{d} V\right)$, the number counts of radio halos with $f \geq f_{\min }(z)$ in a redshift interval, $\Delta z=z_{2}-z_{1}$, is given by

$$
\begin{aligned}
N_{\mathrm{H}}^{\Delta_{z}}\left(>f_{\min }(z)\right)= & \int_{z=z_{1}}^{z=z_{2}} \mathrm{~d} z^{\prime}\left(\frac{\mathrm{d} V}{\mathrm{~d} z^{\prime}}\right) \\
& \int_{P_{\min }\left(f_{\min }^{*} z^{\prime}\right)} \frac{\mathrm{d} N_{\mathrm{H}}\left(P(v), z^{\prime}\right)}{\mathrm{d} P\left(v_{\mathrm{o}}\right) \mathrm{d} V} \cdot \mathrm{d} P\left(v_{\mathrm{o}}\right)
\end{aligned}
$$

In Fig. 8, we show the all-sky number of radio halos with $v_{\mathrm{s}} \geq 120 \mathrm{MHz}$ in different redshift intervals detectable by typical LOFAR surveys of different sensitivities $(0.1 \ldots 1.5 \mathrm{mJy} / \mathrm{beam}$, see figure caption) and following the approaches i) (left panel) and ii) (right panel) described above.

The LOFAR all-sky survey (e.g., Röttgering 2009, priv. comm.) is expected to reach an rms $=0.1 \mathrm{mJy} / \mathrm{beam}$ at $120 \mathrm{MHz}$. Considering the case i) (Fig. 8, left panel) with $\xi \sim 2-3$, we predict that this survey will detect more than 350 radio halos at redshift $\leq 0.6$, in the northern hemisphere $(\delta \geq 0)$ and at high
Galactic latitudes $(|b| \geq 20)$. This will increase the statistics of radio halos by about a factor of 20 with respect to that produced by the NVSS. The LOFAR commissioning MS $^{3}$ survey is expected to reach sensitivities of $\approx 0.5 \mathrm{mJy} / \mathrm{b}$ at $150 \mathrm{MHz}$. Based on our results, $\approx 100$ radio halos are expected to be discovered by this survey within a one year timescale.

The spectral properties of the population of radio halos visible by the future radio surveys at low frequencies are expected to change with the increasing sensitivity of these surveys. In Fig. 8, we show the total number of halos with $v_{\mathrm{s}} \geq 120 \mathrm{MHz}$ (solid lines) and the number of halos with a spectral steepening at low frequencies, $120 \leq v_{\mathrm{s}} \leq 600 \mathrm{MHz}$. The latter class of radio halos has a synchrotron spectral index $\alpha>1.9$ in the range 250$600 \mathrm{MHz}$, and would become visible only at low frequencies, $v_{\mathrm{o}}<600 \mathrm{MHz}$. We find that about $55 \%$ of radio halos in the LOFAR all-sky survey at $120 \mathrm{MHz}$ is expected to belong to this class of ultra-steep spectrum radio halos, while radio halos of higher $v_{\mathrm{s}}$ are expected to dominate the population in shallower surveys. This is simply because, for the reasons explained in 


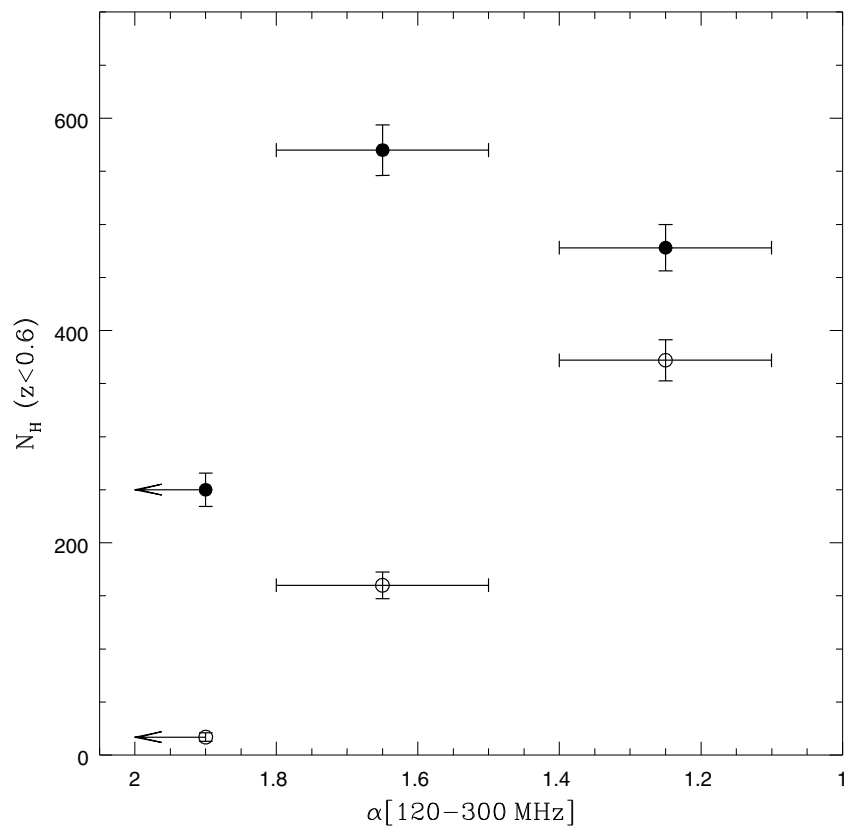

Fig. 9. Spectral index distribution of radio halos, calculated for the range $120-330 \mathrm{MHz}$, for the number counts in Fig. 8 (left panel) and assuming $\xi F=0.1$ (filled symbols) and $0.5 \mathrm{mJy} / \mathrm{b}$ (empty symbols). Spectral indices are calculated for homogeneous models, with parameters given in Sect. 2, assuming the $v_{\mathrm{b}}$ distribution derived from the Monte Carlo synthetic clusters. Radio halos are binned according to the following values of $v_{\mathrm{b}}$ : $v_{\mathrm{b}}>90 \mathrm{MHz}, 35<v_{\mathrm{b}}<90 \mathrm{MHz}$, and $v_{\mathrm{b}}<35 \mathrm{MHz}$ (from right to left). The horizontal bars encompass the variation in $\alpha$ due to the range of $v_{\mathrm{s}} / v_{\mathrm{b}}$ in Fig. 2.

Sect. 2.2, low frequency radio halos are expected to populate the low power-end of the RHLF (e.g., Fig. 5). Complementary information is given in Fig. 9 that shows the expected distribution of halo spectral indices, with reference to the number distributions in Fig. 8, and its evolution with sensitivity of radio observations; the spectra in Fig. 9 have been calculated for the range $120-300 \mathrm{MHz}$ assuming homogeneous models.

Ultra-steep spectrum halos are a unique prediction of the turbulent re-acceleration model (e.g., Brunetti et al. 2008) and our expectations demonstrate the potential of LOFAR to constrain present models for the origin of radio halos.

\subsection{Application to X-ray selected cluster samples}

Although unbiased surveys of radio halos provide an important way to measure the occurrence of these sources (Sect. 3.1), a potential problem with these approaches is the identification of both radio halos and their hosting clusters. This is because radio halos constitute only a very small fraction of the entire radio source population and need to be distinguished from confused regions produced by the superpositions of radio AGNs and starburst galaxies. Alternatively, an efficient approach is to exploit deep LOFAR surveys of X-ray selected samples of galaxy clusters. Here we derive the number of radio halos, and their flux and redshift distributions, that should be detected by LOFAR observations of X-ray selected clusters.

There are several catalogs of X-ray selected clusters in the northern hemisphere that contain clusters extracted from the ROSAT All-Sky Survey (RASS, Trümper 1993). At redshift $\leq 0.3$, the ROSAT Brightest Cluster Sample and its extension to lower X-ray fluxes (eBCS, Ebeling et al. 1998, 2000) and the Northern ROSAT All-Sky (NORAS) Cluster Survey
(Böhringer et al. 2000) provide cluster catalogs with X-ray flux $f_{X[0.1-2.4] \mathrm{keV}} \gtrsim 3 \times 10^{-12} \mathrm{erg} \mathrm{s}^{-1} \mathrm{~cm}^{-2}$; the eBCS is $75 \%$ complete down to this flux limit. The extension of these catalogs to higher redshifts led to the Massive Cluster Survey (MACS, Ebeling et al. 2001), which contains clusters with $f_{X[0.1-2.4] \mathrm{keV}} \geq$ $1 \times 10^{-12} \mathrm{erg} \mathrm{s}^{-1} \mathrm{~cm}^{-2}$ at $z=0.3-0.6$. All these surveys have optical follow-ups and provide a useful starting point for detecting radio halos in LOFAR surveys.

A well-known correlation exists between the synchrotron power of giant radio halos and the X-ray luminosity of the hosting clusters, $P(1.4) \propto L_{\mathrm{x}}^{x}$, where $x \simeq 2$ (e.g., Liang et al. 2000; Bacchi et al. 2003; Enßlin \& Röttgering 2002; Cassano et al. 2006a; Brunetti et al. 2009). This implies that the X-ray flux limit of the survey, $f_{\mathrm{X}}$, is related to the radio flux of halos. The minimum flux of radio halos that can be detected at redshift $z$ is given by the maximum value of the minimum radio flux due to the sensitivity of radio surveys (Sect. 3.1) and that constrained by $f_{\mathrm{X}}$ by the radio - X-ray correlation. To address this issue at $v_{\mathrm{o}}=120 \mathrm{MHz}$ in the case of radio halos with $v_{\mathrm{s}} \geq 1.4 \mathrm{GHz}$, we assume a correlation between the monochromatic radio luminosity at $120 \mathrm{MHz}$ and $L_{\mathrm{X}}$ rescaled from that at $1.4 \mathrm{GHz}$ by means of Eq. (6). For halos with lower $v_{\mathrm{s}}$ (yet $v_{\mathrm{s}}>120 \mathrm{MHz}$ ) the correlation between the radio luminosity at $120 \mathrm{MHz}$ and $L_{\mathrm{X}}$ is obtained from Eq. (8), which accounts for the lower radio power expected for halos with steeper spectra (Sect. 2.2).

In this section, we model the sensitivity of LOFAR at $120 \mathrm{MHz}$ following the approach i) described in Sect. 3 (Eq. (10)). More specifically, to detect radio halos, we consider $120 \mathrm{MHz}$ LOFAR follow-up of a cluster catalog obtained by combining the eBCS (at $z \leq 0.3)$ and the MACS $(0.3<z<0.6)$ samples, and assume reference sensitivities of radio observations of $\xi F=0.25$ and $1 \mathrm{mJy} /$ beam. The minimum $L_{\mathrm{X}}$ of cluster for which these radio observations are expected to detect giant radio halos is evaluated by combining the above radio sensitivity and the minimum $L_{X}$ in cluster catalogs at redshift $z$, and is shown in Fig. 10 by considering different $v_{\mathrm{s}}$ (see figure caption for details). One may note that at intermediate redshift and higher redshift the luminosity-limit is driven by the X-ray flux limit of the eBCS and MACS catalogs, respectively. On the other hand, we expect that in the redshift range where the minimum $L_{X}$ is constrained by the radio sensitivity, radio halos with $v_{\mathrm{s}}$ in the range 120-240 MHz (Fig. 10, black lines) can be detected in clusters of X-ray luminosity about 3 times higher than that of clusters with $v_{\mathrm{s}} \geq 1.4 \mathrm{GHz}$ halos (Fig. 10, magenta lines).

In Fig. 11, we show the cumulative and differential number counts of radio halos expected from the LOFAR follow-up of eBCS and MACS clusters at $120 \mathrm{MHz}$. This is obtained from Eq. (12) and by taking into account both the selection criteria illustrated in Fig. 10 and the sky coverage of the eBCS and MACS surveys. The inflection in the number counts at $z=0.3$ is caused by the change in the X-ray selection criteria (see Fig. 10) between the eBCS $(z \leq 0.3)$ and the MACS $(z \geq 0.3)$ cluster sample. We expect that the LOFAR all-sky survey, with a planned sensitivity in line with the case $\xi F=0.25 \mathrm{mJy} / \mathrm{beam}$ (Fig. 11, upper panels), will discover about 130 radio halos out of the $\sim 400$ clusters in the eBCS and MACS catalogs. Remarkably, about $40 \%$ of these radio halos are expected to have $v_{\mathrm{s}} \leq 600 \mathrm{MHz}$, thus to be halos with extreme steep spectra at $\mathrm{GHz}$ frequencies. The majority of radio halos in eBCS and MACS clusters is expected to be found at $z=0.2-0.4$, while the small number of clusters at $z \geq 0.5$ with X-ray flux above the flux limit of the MACS catalog does not allow a statistically solid expectations, although we may expect a couple of radio halos hosted in MACS clusters at this redshift. At this 


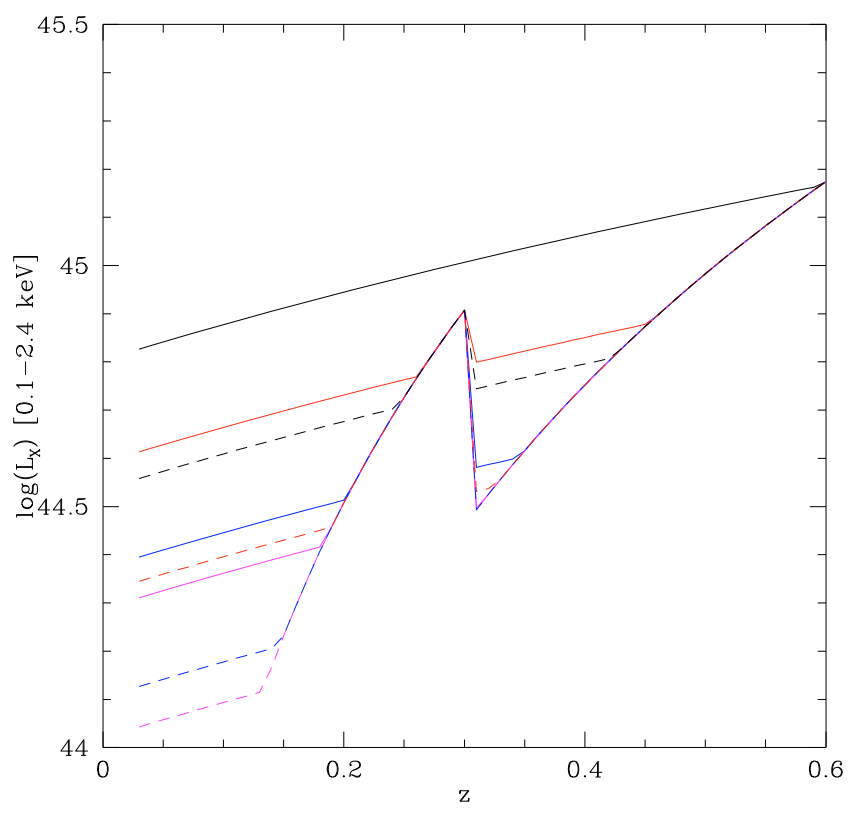

Fig. 10. Minimum X-ray luminosity of clusters with detectable halos at $v_{\mathrm{o}}=120 \mathrm{MHz}$. Calculations are shown for $\xi F=0.25$ (dashed lines) and $1 \mathrm{mJy} / \mathrm{b}$ (solid lines), and for different ranges of $v_{\mathrm{s}}: 120<v_{\mathrm{s}}<$ $240 \mathrm{MHz}$ (black lines), $240<v_{\mathrm{s}}<600 \mathrm{MHz}$ (red lines), $600<v_{\mathrm{s}}<$ $1400 \mathrm{MHz}$ (blue lines) and $v_{\mathrm{s}} \geq 1.4 \mathrm{GHz}$ (magenta lines) (from top to bottom).

redshift, we expect that only major mergers in massive clusters $\left(M_{\mathrm{v}} \geq 2 \times 10^{15} M_{\odot}\right)$ can generate radio halos with $v_{\mathrm{s}} \geq 1.4 \mathrm{GHz}$ (Fig. 4, right panel). The powerful radio halo discovered in the cluster MACS J0717.5 +3745 (e.g., Bonafede et al. 2009; van Weeren et al. 2009) is consistent with these expectations.

Figure 11 (lower panels) shows the expected number counts of radio halos assuming the more conservative case $\xi F=$ $1 \mathrm{mJy} / \mathrm{b}$ that is suitable for exploring the potential of the LOFAR MS ${ }^{3}$ commissioning survey. In this case, about 80 radio halos are expected to be found in eBCS and MACS clusters, and about 20 of these halos are expected to have $v_{\mathrm{S}}<600 \mathrm{MHz}$. We note that the number of radio halos expected to be detected in follow-up observations of eBCS and MACS clusters increases by less than a factor of 2 because of a substantial drop in radio sensitivity from $\xi F=1$ to $0.25 \mathrm{mJy} / \mathrm{b}$. This is not surprising as the majority of radio halos that are expected to be discovered by deep radio observations should be found in galaxy clusters of X-ray luminosity below the luminosity-threshold of the eBCS and MACS catalogs (e.g., Fig. 10).

The eBCS cluster sample contains 300 galaxy clusters at $z<0.3$ and covers the northern hemisphere. The redshift and $\mathrm{X}$-ray luminosity distribution of eBCS clusters is public (Ebeling et al. 1998, 2000) and thus we can provide a more quantitative expectation based on e.g., the more conservative case, $\mathrm{MS}^{3}$-like that assumes that $\xi F=1 \mathrm{mJy} /$ beam at $120 \mathrm{MHz}$ (in this case, the selection function of clusters in the $L_{X}-z$ plane is reported in Fig. 10, solid lines at $z<0.3$ ).

In Fig. 12, we show the distribution of the expected radio halos in the eBCS clusters in two redshift intervals: 0-0.2 and $0.2-0.3$ (left and right panels, respectively). We find that radio observations at $120 \mathrm{MHz}$ are expected to discover radio halos in about 60 clusters, i.e., in about $20 \%$ of eBCS clusters. In addition, about 12 of these halos are expected to have very steep radio spectra, $v_{\mathrm{s}}<600 \mathrm{MHz}$ (magenta, shadowed region in Fig. 12).
Finally, the percentage of clusters with radio halos is expected to increase with the X-ray luminosity of the hosting clusters. This is particularly relevant in the redshift interval $z=$ 0-0.2 when comparing with expectations calculated based on the assumption that the fraction of clusters hosting radio halos is constant with cluster mass (Fig. 12 dashed lines, see caption). Consequently, LOFAR will be able to readily test this unique expectation of the turbulent re-acceleration model.

\section{Summary and conclusions}

We have performed Monte Carlo simulations to model the formation and evolution of giant radio halos in the framework of the merger-induced particle acceleration scenario (see Sect. 2). Following Cassano et al. (2006a), we have used homogeneous models that assume a) an average value of the magnetic field strength in the radio halo volume that scales with cluster mass as $B=B_{\langle M\rangle} M_{\mathrm{v}}^{b}$; and b) that a fraction, $\eta_{t}$, of the $P \mathrm{~d} V$ work done by subclusters crossing the main clusters during mergers goes into magneto-acoustic turbulence. Although simple, these models reproduce the presently observed fraction of galaxy clusters with radio halos and the scalings between the monochromatic radio power of halos at $1.4 \mathrm{GHz}$ and the mass and X-ray luminosity of the host clusters (e.g., Cassano et al. 2006a, 2008; Venturi et al. 2008), provided that the model parameters $\left(B_{\langle M\rangle}, b, \eta_{t}\right)$ lie within a fairly constrained range of values (Fig. 7 in Cassano et al. 2006a); in the present paper, we have adopted a reference set of parameters, i.e., $\langle B\rangle=1.9 \mu \mathrm{G}, b=1.5, \eta_{t}=0.2$, that fall in that range.

In Fig. 6, we show that the expected number counts of giant radio halos at $v_{\mathrm{O}}=1.4 \mathrm{GHz}$ obtained with this set of parameters are in good agreement with both the data at low redshift (NVSS-XBACS selected radio halos, Giovannini et al. 1999) and intermediate redshift (clusters in the "GMRT radio halo survey", Venturi et al. 2007, 2008).

The most important expectation of the turbulent reacceleration scenario is that the synchrotron spectrum of radio halos should become gradually steeper above a frequency, $v_{\mathrm{S}}$, that is determined by the energetics of the merger events that generate the halos and by the electron radiative losses (e.g., Fujita et al. 2003; Cassano \& Brunetti 2005). Consequently, the population of radio halos is expected to consist of a mixture of halos with different spectra, steep-spectrum halos being more common in the Universe than those with flatter spectra (e.g., Cassano et al. 2006a). The discovery of these very steep-spectrum halos will allow us to test the above theoretical conjectures.

In Sect. 2, we have derived the expected radio halo luminosity functions (RHLF) at frequency $v_{\mathrm{o}}$ that account for the contributions of the different populations of radio halos with $v_{\mathrm{s}} \geq v_{\mathrm{o}}$. The RHLF are obtained combining the theoretical mass function of radio halos (of different $v_{\mathrm{s}} \geq v_{\mathrm{O}}$ ) with the radio power-cluster mass correlation (Eq. (4)). The expected monochromatic radio power at $v_{\mathrm{o}}$ of halos hosted by clusters with mass $M_{\mathrm{v}}$ is extrapolated from the observed $P(1.4)-M_{\mathrm{v}}$ correlation by assuming simple scaling relations, appropriate for homogeneous models, that account for the dependence of the emitted synchrotron power on $v_{\mathrm{S}}$ (Eqs. (8), (9)).

As a relevant case, we calculate the expected RHLF at $v_{\mathrm{o}}=120 \mathrm{MHz}$ (Fig. 5). The shape of the RHLF can be approximated by a power law over more than two orders of magnitude in radio power. Homogeneous models imply that scalings 

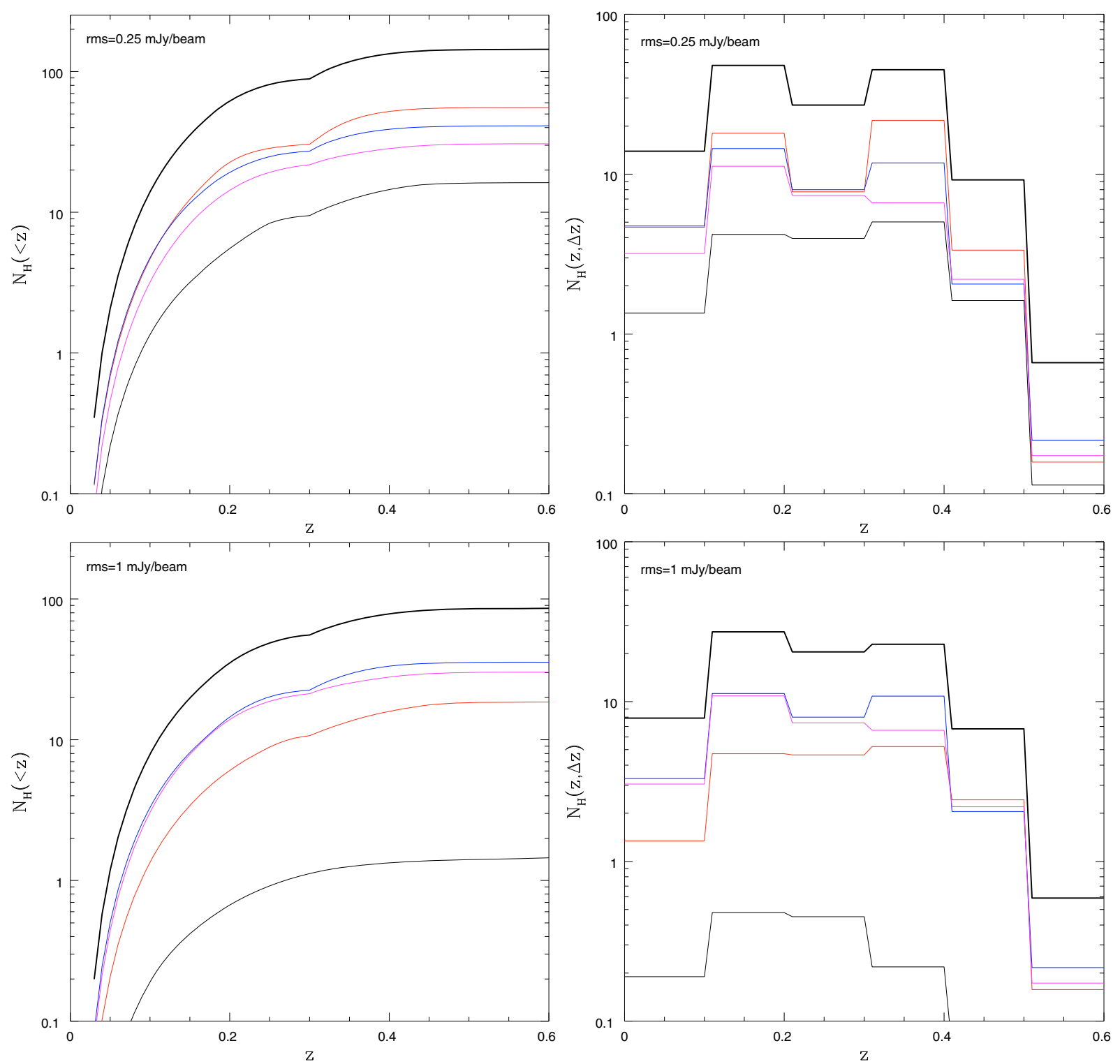

Fig. 11. Integrated (left) and differential (right) number counts of radio halos from radio follow up of eBCS and MACS clusters (see text). Calculations are shown for $\xi F=0.25$ (upper panels) and $1.0 \mathrm{mJy} / \mathrm{b}$ (lower panels) at $120 \mathrm{MHz}$. Thick (black) solid lines give the case $v_{\mathrm{s}} \geq$ $120 \mathrm{MHz}$, while differential contributions are shown with different colors: $v_{\mathrm{s}} \geq 1.4 \mathrm{GHz}$ (magenta lines), $600<v_{\mathrm{s}}<1400 \mathrm{MHz}$ (blue lines), $240<v_{\mathrm{s}}<600 \mathrm{MHz}$ (red lines) and $120<v_{\mathrm{s}}<240 \mathrm{MHz}$ (black thin lines).

between $v_{\mathrm{s}}$, cluster mass and the radio luminosity at $v_{\mathrm{o}}, P_{v_{\mathrm{s}}}\left(v_{\mathrm{o}}\right)$ are given by

$v_{\mathrm{s}} \propto M^{4 / 3+b} \frac{(1+\Delta M / M)^{3}}{\left(\langle B\rangle^{2}+B_{\mathrm{cmb}}^{2}\right)^{2}}$,

and from Eq. (9) and the $P_{1.4}-M_{\mathrm{v}}$ correlation

$P_{v_{\mathrm{s}}}\left(v_{\mathrm{o}}\right) \propto M_{\mathrm{v}}^{3} v_{\mathrm{s}}^{\alpha}$,

i.e., radio halos with higher $v_{\mathrm{s}}$ are typically generated in massive clusters that undergo major mergers and contribute to the RHLF at higher powers. On the other hand, halos with lower $v_{\mathrm{s}}$ are typically generated in less massive systems and contribute to the RHLF at fainter powers. Radio halos with $v_{\mathrm{s}} \geq 120 \mathrm{MHz}$, however, become increasingly rare in clusters of mass $\leq 5 \times 10^{14} M_{\odot}$ explaining the drop in the RHLF at lower radio powers in Fig. 5. At the same time, halos with monochromatic radio emission at $120 \mathrm{MHz}>10^{26} \mathrm{~W} \mathrm{~Hz}^{-1}$ would be generated by very energetic merging events in very massive clusters, which are extremely rare, and this explains the RHLF cut-off at higher synchrotron powers in Fig. 5.

In Sect. 3, we discussed the expected number counts of radio halos at $120 \mathrm{MHz}$ that would allow us to explore most effectively the potential of upcoming LOFAR surveys in constraining present models.

A crucial step in this analysis is the estimate of the minimum diffuse flux from giant radio halos that is detectable by these surveys. Because the LOFAR capabilities will become clearer during the upcoming commissioning phase, we exploit two complementary approaches: i) we required that at least half of the radio halo emission is above a fixed brightness-threshold, $\xi F$ ( $F$ being the rms of LOFAR surveys; ii) we required that the signal from the radio halo is $\geq 3 \times \xi F$ in at least 5 beam areas of LOFAR observations. In both cases we assume that the radial profile of radio halos has a fixed shape calibrated by means of several well studied halos at $1.4 \mathrm{GHz}$, which introduces a potential source of uncertainty. 
R. Cassano et al.: Unveiling radio halos in galaxy clusters in the LOFAR era
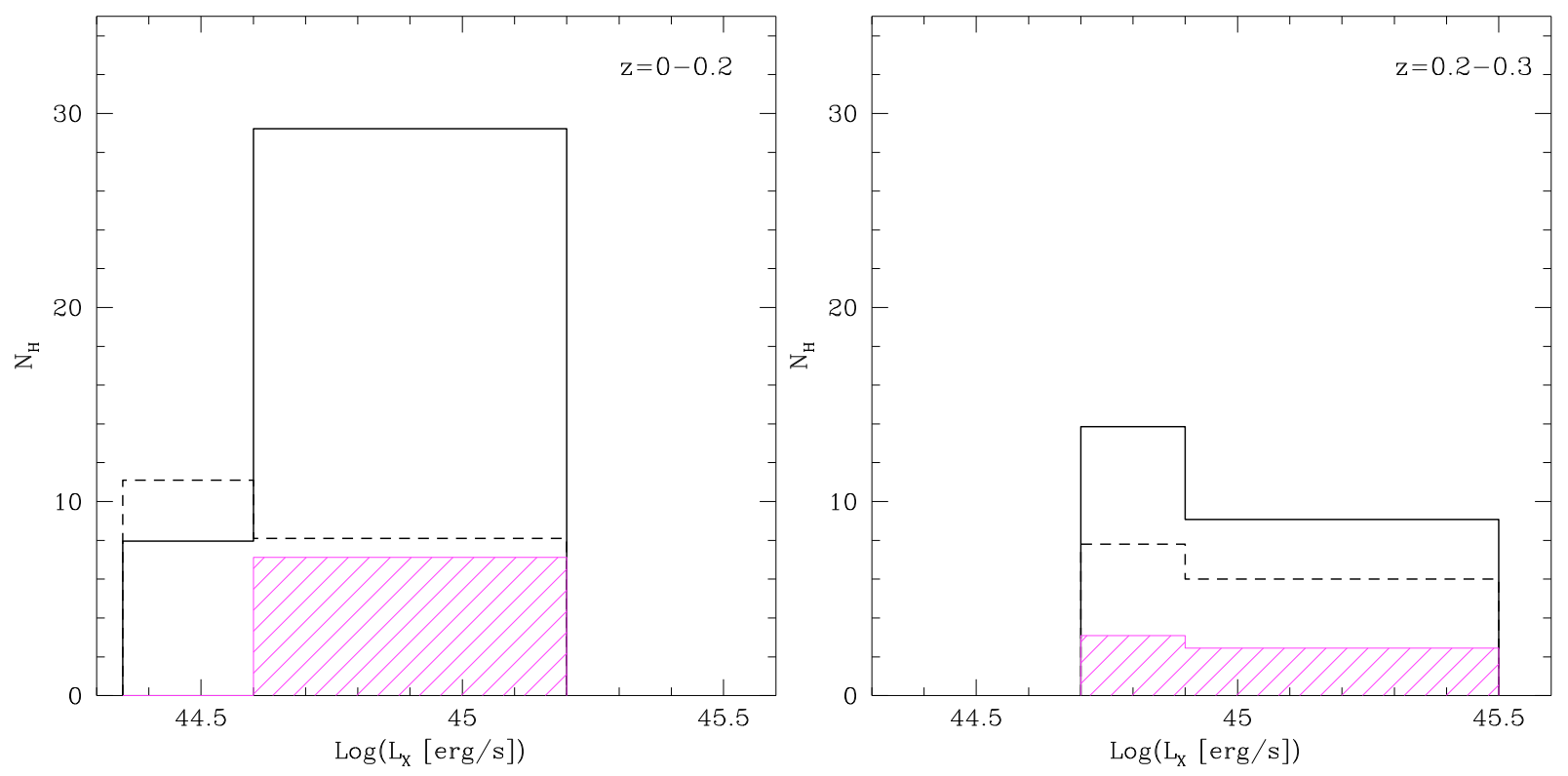

Fig. 12. Number distributions of radio halos in eBCS clusters from radio follow-up are shown in different X-ray luminosity bins of the hosting clusters and in two redshift bins : 0-0.2 (left panel) and 0.2-0.3 (right panel). Calculations are obtained assuming a sensitivity at $120 \mathrm{MHz}$, $\xi F=1 \mathrm{mJy} / \mathrm{b}$ and beam $=25 \times 25$ arcsec. Solid lines show the number of radio halos with $v_{\mathrm{s}} \geq 120 \mathrm{MHz}$, while the dashed areas mark the contribution from halos with $120<v_{\mathrm{s}}<600 \mathrm{MHz}$. For comparison, the dashed lines show the expectations assuming that a fixed fraction of galaxy clusters $=30 \%$ host radio halos and that all radio halos have $v_{\mathrm{s}} \geq 1400 \mathrm{MHz}$.

Despite the uncertainties caused by the unavoidable simplifications in our calculations, the expected number counts of radio halos highlights the potential of future LOFAR surveys. By assuming the expected sensitivity of the LOFAR all-sky survey (e.g., Röttgering 2009; priv. comm.), rms $=0.1 \mathrm{mJy} / \mathrm{b}$, and $\xi \sim 2-3$, we predict that about 350 giant radio halos $(\sim 200$ considering the case ii)) can be detected at redshift $\leq 0.6$. This means that LOFAR will increase the statistics of these sources by a factor of $\sim 20$ with respect to present-day surveys. About $55 \%$ of these halos are predicted with a synchrotron spectral index $\alpha>1.9$ in the range $250-600 \mathrm{MHz}$, and would brighten only at lower frequencies, which are inaccessible to present observations. Most important, the spectral properties of the population of radio halos are expected to change with the increasing sensitivity of the surveys as steep spectrum radio halos are expected to populate the low-power end of the RHLF. A large fraction of radio halos with spectra steeper than $\alpha \approx 1.5$ (e.g., Fig. 9) is expected to allow a robust discrimination between different models of radio halos, for instance in this case simple energetic arguments would exclude a secondary origin of the emitting electrons (e.g., Brunetti 2004; Brunetti et al. 2008).

Because of the large number of expected radio halos, a potential problem with these surveys is the identification of halos and their hosting clusters. As a matter of fact, we expect that LOFAR surveys will detect radio halos in galaxy clusters with masses $\gtrsim 6-7 \times 10^{14} M_{\odot}$ at intermediate redshift. On the other hand, statistical samples of X-ray selected clusters, which are unique tools for identifying the hosting clusters, typically select more massive clusters at intermediate $z$. Consequently, we explored the potential of the first LOFAR surveys as deep followups of available X-ray selected samples of galaxy clusters.

We calculate the radio halo number counts expected from the follow-up of clusters in the eBCS and MACS samples which contain $\sim 400$ galaxy clusters in the redshift range $0-0.6$. We expect that the LOFAR all-sky survey, with a planned sensitivity in line with $\xi F=0.25 \mathrm{mJy} / \mathrm{b}$, will discover about 130 radio halos in eBCS and MACS clusters and that about $40 \%$ of these radio halos will have a very steep spectrum, with $v_{\mathrm{s}} \leq 600 \mathrm{MHz}$. The majority of radio halos in eBCS and MACS clusters are expected to be at $z=0.2-0.4$, while the small number of clusters at $z \geq 0.5$ in the MACS catalog does not allow us to form a statistically solid expectations, although we expect a couple of radio halos to be hosted by MACS clusters at this redshift.

The $\mathrm{MS}^{3}$ survey will be carried out in 2010 , covering the northern hemisphere, and is expected to reach a noise level of about $0.5 \mathrm{mJy} / \mathrm{b}$ at $150 \mathrm{MHz}$, implying a sensitivity to diffuse emission from galaxy clusters of about one order of magnitude (assuming $\alpha \approx 1.3$ ) higher than present surveys (e.g., NVSS, Condon et al. 1998; VLSS, Cohen et al. 2007, WENSS, Rengelink et al. 1997).

We considered $\mathrm{MS}^{3}$ pointings towards the fields of the about 300 galaxy clusters at $z \leq 0.3$ in the eBCS catalogs. We found that about 60 radio halos are expected to be detected by $\mathrm{MS}^{3}$ observations in these clusters, $25 \%$ of them (10-15 halos) with $v_{\mathrm{s}} \leq 600 \mathrm{MHz}$. Fairly sensitive GMRT observations of eBCS clusters at redshift $0.2-0.3$ are already available (Venturi et al. 2007, 2008), and in a few cases we expect that radio halos would be detectable in the $\mathrm{MS}^{3}$ images, where no diffuse radio emission is detected at $610 \mathrm{MHz}$. We also find that $\mathrm{MS}^{3}$ observations of eBCS clusters at $z=0-0.2$ can be used to test the increase in the fraction of cluster with radio halos, with the X-ray luminosity of the host clusters, which is a unique prediction of our model (Fig. 12).

The most important simplification of our calculations is the use of homogeneous models. Non-homogeneous approaches, which model the spatial dependence of the acceleration efficiency and magnetic field in the halo volume (e.g., Brunetti et al. 2004), and possibly their combination with future numerical simulations, will provide an additional step in interpreting LOFAR data. Also the use of the extended PS theory is expected to introduce some biases. For instance, it is well-known that the PS mass function underpredicts the expected number of 
massive clusters $\left(M>10^{15} M_{\odot}\right)$ at higher redshift, $z \sim 0.4-0.5$, by a factor of $\sim 2$ with respect to that found in $N$-body simulations (e.g., Governato et al. 1999; Bode et al. 2001; Jenkins et al. 2001). In our model since the vast majority of halos at these redshift is associated with massive clusters, the use of the PS mass function implies that the RHNC at $z>0.4-0.5$ could be underestimated by a similar factor. A refinement of the approach proposed in the present paper could be achieved by using galaxy cluster merger trees extracted from $\mathrm{N}$-body simulations. These would also allow a more realistic description of the merger events (e.g., spatially resolved, multiple mergers).

In the present paper, we focus on a reference set of model parameters. Cassano et al. (2006a) discussed the dependence of model expectations at $1.4 \mathrm{GHz}$ on these parameters. Based on their analysis, we expect that all the general results given in the present paper are independent of the adopted parameter values. The expected number counts of halos should change by a factor of $\sim 2-2.5$ considering sets of model parameters within the region $\left(\langle B\rangle, b, \eta_{t}\right)$ that allow us to reproduce the observed $P_{1.4}-M_{\mathrm{v}}$ correlation. In this case, the number of halos that we expect decreases between super-linear sets of parameters $(b>1$ and $\langle B\rangle \geq 1.5 \mu \mathrm{G})$ and sub-linear cases $(b<1$ and $\langle B\rangle \leq 1.5 \mu \mathrm{G})$ (see also Fig. 4 in Cassano et al. 2006b); a more detailed study will be presented in a future paper.

Acknowledgements. We thank the anonymous referee for useful comments. This work is partially supported by ASI and INAF under grants PRIN-INAF 2007, PRIN-INAF 2008 and ASI-INAF I/088/06/0.

\section{References}

Bacchi, M., Feretti, L., Giovannini, G., et al. 2003, A\&A, 400, 465 Blasi, P., 2001, APh, 15, 223

Blasi, P., \& Colafrancesco, S. 1999, Astropart. Phys., 12, 169 Bode, P., Bahcall, N. A., Ford, E. B. et al. 2001, ApJ, 551, 15 Bonafede, A., Feretti, L., Giovannini, G., et al. 2009, A\&A, 503, 707 Böhringer, H., Voges, W., Huchra, J. P., et al. 2000, ApJS, 129, 435 Böhringer, H., Schuecker, P., Guzzo, L., et al. 2004, A\&A, 425, 367 Brunetti, G. 2004, JKAS, 37, 493

Brunetti, G., \& Lazarian, A. 2007, MNRAS, 378, 245

Brunetti, G., Setti, G., Feretti, L., et al. 2001, MNRAS, 320, 365

Brunetti, G., Blasi, P., Cassano, R., et al. 2004, MNRAS, 350, 1174

Brunetti, G., Venturi, T., \& Dallacasa, D., et al. 2007, ApJ, 670, 5

Brunetti, G., Giacintucci, S., Cassano, R., et al. 2008, Nature, 455, 944

Brunetti, C., Cassano, R., Dolag, K., \& Setti, G. 2009, A\&A, 507, 661

Brüggen, M., Ruszkowski, M., Simionescu, A., et al. 2005, ApJ, 631, L21

Buote, D. A. 2001, ApJ, 553, 15

Cassano, R., \& Brunetti, G. 2005, MNRAS 357, 1313
Cassano, R., Brunetti, G., \& Setti, G. 2006a, MNRAS 369,1577

Cassano, R., Brunetti, G., \& Setti, G. 2006b, Astr. Nachr. 327, 557

Cassano, R., Brunetti, G., \& Venturi, T. 2008, et al. A\&A, 480, 687

Cohen, A. S., Lane, W. M., Cotton, W. D., et al. 2007, AJ, 134, 1245

Condon, J. J., Cotton, W. D., Greisen, E. W., et al. 1998, AJ, 115, 1693

Dallacasa, D., Brunetti, G., Giacintucci, S., et al. 2009, ApJ, 699, 1288

Dennison, B. 1980, ApJ, 239

Dolag, K., Bartelmann, M., \& Lesch, H. 2002, A\&A, 387, 383

Ebeling, H., Voges, W., Bohringer, H., et al. 1996, MNRAS, 281, 799

Ebeling, H., Edge, A. C., Böhringer, H., et al. 1998, MNRAS, 301, 881

Ebeling, H., Edge, A. C., Allen, S. W., et al. 2000, MNRAS, 318, 333

Ebeling, H., Edge, A. C., \& Henry, J. P. 2001, ApJ, 553, 668

Ellingson, S. W., Clarke, T. E., Cohen, A., et al. 2009, Proc. of the IEEE, 97, Issue 8, 1421

Enßlin, T. A., Biermann, P. L., Klein, U., et al. 1998, A\&A, 332, 395

Enßlin, T. A., \& Röttgering, H. 2002, A\&A, 396, 83

Feretti, L. 2005, in X-Ray and Radio Connections, published electronically by NRAO, ed. L.O. Sjouwerman, \& K. K. Dyer

Ferrari, C., Govoni, F., Schindler, S., Bykov, A. M., \& Rephaeli, Y. 2008, Space Sci. Rev., 134, 93

Fujita, Y., Takizawa, M., \& Sarazin, C. L. 2003, ApJ 584, 190

Giovannini, G., Tordi, M., \& Feretti, L. 1999, NewA 4, 141

Governato, F., Babul, A., Quinn, T., et al. 1999, MNRAS, 307, 949

Govoni, F., Feretti, L., Giovannini, G., et al. 2001, A\&A, 376, 803

Govoni, F., Markevitch, M., Vikhlinin, A., et al. 2004, ApJ, 605, 695

Hwang C.-Y. 2004, JKAS, 37, 461

Hoeft, M., \& Brüggen, M. 2007, MNRAS, 375, 77

Hoeft, M., Brüggen, M., Yepes, G., Gottlber, S., \& Schwope, A. 2008, MNRAS, 391,1511

Jenkins, A., Frenk, C. S., White, S. D. M., et al. 2001, MNRAS, 321, 372

Liang, H., Hunstead, R. W., Birkinshaw, M., et al. 2000, ApJ, 544, 686

Lacey, C., \& Cole, S. 1993, MNRAS, 262, 627

Miniati, F., Jones, T. W., Kang, H., et al. 2001, ApJ, 562, 233

Petrosian, V. 2001, ApJ, 557, 560

Pfrommer, C., Enßlin, T. A., \& Springel, V. 2008, MNRAS, 385, 1211

Press, W. H., \& Schechter, P. 1974, ApJ, 187, 425

Rengelink, R. B., Tang, Y., de Bruyn, A. G., et al. 1997, A\&AS, 124, 259

Röttgering, H. J. A., Braun, R., Barthel, P. D., et al. 2006, proceedings of the conference Cosmology, galaxy formation and astroparticle physics on the pathway to the SKA, Oxford, April 10-12 [astro-ph/0610596]

Ryu, D., Kang, H., Hallman, E., et al. 2003, ApJ, 593, 599

Ryu, D., Kang, H., Cho, J., et al. 2008, Science, 320, 909

Sarazin, C. L. 1999, ApJ, 520, 529

Schlickeiser, R., Sievers, A., Thiemann, H. 1987, A\&A, 182, 21

Schlickeiser, R., \& Miller, J. A. 1998, ApJ, 492, 352

Schuecker, P., Böhringer, H., Reiprich, T. H., et al. 2001, A\&A, 378, 408

Springel, V., White, S. D. M., \& Jenkins, A. 2005, Nature, 435, 629

Subramanian, K., Shukurov, A., \& Haugen, N. E. L. 2006, MNRAS, 366, 1437

Thierbach, M., Klein, U., \& Wielebinski, R. 2003, A\&A, 397, 53

Trümper, J., 1993, Science, 260, 1769

Venturi, T., Giacintucci, S., Brunetti, G., et al. 2007, A\&A, 463, 937

Venturi, T., Giacintucci, S., Dallacasa, et al. 2008, A\&A, 484, 327

van Weeren, R. J., Röttgering, H. J. A., Bruggen M, et al. 2009, A\&A, 505, 991 